PHYSICAL REVIEW D 89, 054502 (2014)

\title{
Scattering lengths for two pseudoscalar meson systems
}

\author{
Kiyoshi Sasaki, ${ }^{1}$ Naruhito Ishizuka, ${ }^{2,3}$ Makoto Oka, ${ }^{1}$ Takeshi Yamazaki ${ }^{4}$ \\ (PACS-CS Collaboration)
}

\author{
${ }^{1}$ Department of Physics, Tokyo Institute of Technology, Tokyo 152-8551, Japan \\ ${ }^{2}$ Graduate School of Pure and Applied Sciences, University of Tsukuba, Tsukuba, Ibaraki 305-8571, Japan \\ ${ }^{3}$ Center for Computational Science, University of Tsukuba, Tsukuba, Ibaraki 305-8577, Japan \\ ${ }^{4}$ Kobayashi-Maskawa Institute for the Origin of Particles and the Universe, Nagoya University, Nagoya, \\ Aichi 464-8602, Japan
}

(Received 2 December 2013; published 12 March 2014)

\begin{abstract}
Scattering lengths for two pseudoscalar meson systems, $\pi \pi(I=2), K K(I=1)$ and $\pi K(I=3 / 2,1 / 2)$, are calculated from lattice QCD by using the finite size formula. We perform the calculation with $N_{f}=$ $2+1$ gauge configurations generated on $32^{3} \times 64$ lattice using the Iwasaki gauge action and a nonperturbatively $\mathcal{O}(a)$-improved Wilson action at $a^{-1}=2.19 \mathrm{GeV}$. The quark masses correspond to $m_{\pi}=0.17-0.71 \mathrm{GeV}$. For the $\pi K(I=1 / 2)$ system, we use the variational method with the two operators, $\bar{s} u$ and $\pi K$, to separate the contamination from the higher states. In order to obtain the scattering length at the physical quark mass, we fit our results at several quark masses with the formula of the $\mathcal{O}\left(p^{4}\right)$ chiral perturbation theory and that includes the effects of the discretization error from the Wilson fermion, Wilson chiral perturbation theory. We found that the mass dependence of our results near $m_{\pi}=0.17 \mathrm{GeV}$ are described well by Wilson chiral perturbation theory but not by chiral perturbation theory. The scattering lengths at the physical point are given as $a_{0}^{(2)} m_{\pi}=-0.04263(22)(41), a_{0}^{(1)} m_{K}=-0.310(17)(32)$, $a_{0}^{(3 / 2)} \mu_{\pi K}=-0.0469(24)(20)$, and $a_{0}^{(1 / 2)} \mu_{\pi K}=0.142(14)(27)$. Possible systematic errors are also discussed.
\end{abstract}

DOI: $10.1103 /$ PhysRevD.89.054502

PACS numbers: $12.38 . \mathrm{Gc}, 11.15 . \mathrm{Ha}$

\section{INTRODUCTION}

The scattering length is a key quantity for understanding the basic properties of the hadron interaction at low energy. The lattice QCD calculations of the scattering length for many scattering systems have been reported in the past year. Most of the calculations employ the finite-volume method of Lüscher [1], in which the scattering phase shift is related to the energy eigenvalue on a finite volume. In the present work, we consider giving a lattice QCD calculation on the scattering lengths for the $\pi \pi(I=2), K K(I=1)$, $\pi K(I=3 / 2)$, and $\pi K(I=1 / 2)$ systems.

The $S$-wave $\pi \pi$ system has two isospin channels $(I=0$, $2)$. For the $\pi \pi(I=0)$ system, the time correlation function has a disconnected quark diagram. The statistical error of this diagram is very large and it makes a calculation of the scattering length very difficult [2-5]. In the present work, we do not study this channel. For the $\pi \pi(I=2)$ system, whose interaction is experimentally known to be repulsive, after pioneering works with the quenched approximation [2,6-9], several authors reported the realistic calculations with the various formulations of the dynamical fermion [3-5,10-14]. For the $S$-wave $K K(I=1)$ system, only one calculation has been reported by the NPLQCD Collaboration [15]. The $S$-wave $\pi K$ system has two isospin channels $(I=1 / 2,3 / 2)$. For $I=3 / 2$, the interaction is experimentally known to be repulsive. After working with the quenched approximation [16,17], several calculations with dynamical quarks were reported in Refs. [18-20]. For $I=1 / 2$, the interaction is known to be attractive, and existence of a scalar resonance with a broad width is suggested. The NPLQCD Collaboration evaluated the scattering length by using the chiral perturbation theory with the low energy constants (LECs) obtained from the lattice calculations of the decay constants $f_{\pi}$ and $f_{K}$, and the scattering length for the $\pi K(I=3 / 2)$ system [18]. After this work, the direct calculations of the $I=1 / 2$ scattering length have been reported by some groups $[17,19,20]$.

Here, we note that all above calculations of the scattering length were performed in the quark-mass range $m_{\pi} \geq 0.24 \mathrm{GeV}$. A calculation near the physical quark mass is desired to evaluate the reliable results at the physical quark mass. In the present work, we calculate the scattering lengths for the $\pi \pi(I=2), K K(I=1)$, $\pi K(I=3 / 2)$, and $\pi K(I=1 / 2)$ systems in $m_{\pi}=$ $0.17-0.71 \mathrm{GeV}$. For the $\pi K(I=1 / 2)$ system, where the existence of a scalar resonance is suggested, the contamination from the higher states might be non-negligible. To separate the contamination, we use the variational method with the two operators, $\bar{s} u$ and $\pi K$. In order to obtain the scattering length at the physical quark mass, we 
fit our results at several quark masses with the formula of the $\mathcal{O}\left(p^{4}\right)$ chiral perturbation theory (ChPT) and that includes the effects of the discretization error from the Wilson fermion, Wilson chiral perturbation theory (WChPT). We found that the mass dependence of our results near $m_{\pi}=0.17 \mathrm{GeV}$ can be described well by WChPT, but not by ChPT.

This article is organized as follows. In Sec. II, we give the brief description for the Lüscher's finite size formula and the calculation method of the time correlation function. We also give the simulation parameters. In Sec. III, we show our results of the scattering length at several quark masses. In Sec. IV, we discuss the quark-mass dependence of our scattering lengths by using the $\mathcal{O}\left(p^{4}\right)$ ChPT and WChPT. In Sec. V, we evaluate the scattering lengths at the physical point and discuss the possible systematic errors. In Sec. VI, our conclusions are given. All calculations of the present study have been done on the super parallel computers, PACS-CS and T2K-Tsukuba at the University of Tsukuba, and TSUBAME at the Tokyo Institute of Technology. The preliminary results of the present work have been reported in Ref. [21].

\section{METHOD OF CALCULATION}

\section{A. Scattering length}

The $S$-wave scattering phase shift $\delta_{0}$ for the system of two spinless particles with masses $m_{1}$ and $m_{2}$ is related to the energy eigenvalue on the finite volume by

$$
\left[\tan \delta_{0}(k) / k\right]^{-1}=\sqrt{4 \pi} g_{00}(k ; 1),
$$

where $k$ is the scattering momentum related to the energy by $E=\sqrt{m_{1}^{2}+k^{2}}+\sqrt{m_{2}^{2}+k^{2}}$. The function $g_{00}(k ; 1)$ is given by the analytic continuation of

$$
g_{00}(k ; z)=\frac{\sqrt{4 \pi}}{L^{3}} \sum_{\mathbf{p}=2 \pi \mathbf{n} / L}\left(\mathbf{p}^{2}-k^{2}\right)^{-z} \quad\left(\mathbf{n} \in \mathbb{Z}^{3}\right),
$$

which is defined for $\operatorname{Re}(z)>3 / 2$, where $L$ is the spatial extent. The scattering length is defined as

$$
a_{0} \equiv \lim _{k \rightarrow 0} \tan \delta_{0}(k) / k \text {. }
$$

In the case of an attractive interaction on a finite volume, the lowest energy state has a negative $k^{2}$, so that $k$ is pure imaginary. In this case, for $L \rightarrow \infty$, two situations can be considered as

(a) $k^{2} \rightarrow-\kappa^{2}(\kappa \in \mathbb{R})$,

(b) $k^{2} \rightarrow 0$.

In (a), the system has a bound state whose binding momentum is $\kappa$. The $S$ matrix

$$
S=\mathrm{e}^{2 i \delta_{0}(k)}=\frac{i-\tan \delta_{0}(k)}{i+\tan \delta_{0}(k)}
$$

has a pole at $k^{2}=-\kappa^{2}$, and $\tan \delta_{0}(k)$ converges to $-i$ in $L \rightarrow \infty[22,23]$. In (b), the system has no bound state, but only scattering states. In general, if the system has no bound state, we can obtain the scattering length $a_{0}$ by substituting $k$ of the lowest energy state into Eq. (1) and extrapolating it to $L \rightarrow \infty$ regardless of whether the interaction is attractive or repulsive.

\section{B. Time correlation function}

For the $\pi \pi(I=2)$ system, we extract the energy $E$ from the time correlation function

$$
G^{(2)}(t)=\left\langle 0\left|\Omega^{(2)}\left(t_{1}, t\right) \bar{\Omega}^{(2) \dagger}\left(t_{0}\right)\right| 0\right\rangle .
$$

The operators $\Omega^{(2)}$ and $\bar{\Omega}^{(2)}$ are defined by

$$
\begin{aligned}
\Omega^{(2)}\left(t_{1}, t\right) & =\pi^{+}\left(t_{1}\right) \pi^{+}(t) \cdot \mathrm{e}^{m_{\pi}\left(t_{1}-t\right)}, \\
\bar{\Omega}^{(2)}\left(t_{0}\right) & =W_{\pi^{+}}\left(t_{0}+1\right) W_{\pi^{+}}\left(t_{0}\right),
\end{aligned}
$$

where $\pi^{+}(t)$ is the local operator for the $\pi^{+}$meson at the time slice $t$ with the zero spatial momentum and $W_{\pi^{+}}(t)$ is the wall-source operator at the time slice $t$. In Eq. (6), the time slice of one of the wall-source operator is shifted from the time slice of another source operator $t_{0}$ to avoid the Fierz mixing of the wall-source operators [2]. In the previous calculations, the time slice of the sink operators are set at $t_{1}=t$, and they simultaneously run over the whole time extent. We call this calculation method "method I" in the following. We also employ an another method, where the time slice of one of the pions at $t_{1}$ is fixed and only $t$ runs over the whole time extent. This method is called "method II" in the following. In method II, we need to set $t_{1} \gg t$ to avoid contamination from higher energy states produced by the operator at $t_{1}$. For $t_{0} \ll t \ll t_{1}$, $G^{(2)}(t)$ can be written as

$$
G^{(2)}(t)=\sum_{n}\left\langle 0\left|\pi^{+}\right| \pi\right\rangle\left\langle\pi\left|\pi^{+}\right| E_{n}\right\rangle\left\langle E_{n}\left|\bar{\Omega}^{(2) \dagger}\right| 0\right\rangle \cdot \mathrm{e}^{-E_{n}\left(t-t_{0}\right)},
$$

where $|\pi\rangle$ is the pion state and where $\left|E_{n}\right\rangle$ is the $n$th energy eigenstate of the two-pion state with the energy eigenvalue $E_{n}$. The exponential factor $\mathrm{e}^{m_{\pi}\left(t_{1}-t\right)}$ in the definition of $\Omega^{(2)}\left(t_{1}, t\right)$ in Eq. (6) is introduced so that the operator $\Omega^{(2)}\left(t_{1}, t\right)$ has the same time behavior as that of the usual Heisenberg operator, i.e.,

$$
\langle 0| \Omega^{(2)}\left(t_{1}, t\right)=\langle 0| \Omega^{(2)}\left(t_{1}, t_{2}\right) \mathrm{e}^{-H\left(t-t_{2}\right)} \text { for } t_{1} \gg t, t_{2},
$$

with the Hamiltonian $H$.

For the $K K(I=1)$ system, we similarly extract $E$ from the time correlation functions 


$$
G^{(1)}(t)=\left\langle 0\left|\Omega^{(1)}\left(t_{1}, t\right) \overline{\mathbf{\Omega}}^{(1) \dagger}\left(t_{0}\right)\right| 0\right\rangle .
$$

$\Omega^{(1)}$ and $\bar{\Omega}^{(1)}$ are defined by

$$
\begin{aligned}
\Omega^{(1)}\left(t_{1}, t\right) & =K^{+}\left(t_{1}\right) K^{+}(t) \cdot \mathrm{e}^{m_{K}\left(t_{1}-t\right)}, \\
\bar{\Omega}^{(1)}\left(t_{0}\right) & =W_{K^{+}}\left(t_{0}+1\right) W_{K^{+}}\left(t_{0}\right),
\end{aligned}
$$

where $K^{+}(t)$ is the local operator for the $K^{+}$meson at the time slice $t$ with the zero spatial momentum and $W_{K^{+}}(t)$ is the wall-source operator at the time slice $t$.

Also for the $\pi K(I=3 / 2)$ system, we define the time correlation function

$$
G^{(3 / 2)}(t)=\left\langle 0\left|\Omega^{(3 / 2)}\left(t_{1}, t\right) \bar{\Omega}^{(3 / 2) \dagger}\left(t_{0}\right)\right| 0\right\rangle,
$$

where $\Omega^{(3 / 2)}$ and $\bar{\Omega}^{(3 / 2)}$ are defined by

$$
\begin{aligned}
\Omega^{(3 / 2)}\left(t_{1}, t\right) & =K^{+}\left(t_{1}\right) \pi^{+}(t) \cdot \mathrm{e}^{m_{K}\left(t_{1}-t\right)}, \\
\bar{\Omega}^{(3 / 2)}\left(t_{0}\right) & =W_{K^{+}}\left(t_{0}+1\right) W_{\pi^{+}}\left(t_{0}\right) .
\end{aligned}
$$

Next, we construct the time correlation function of the $\pi K(I=1 / 2)$ system. If a resonance state exists and its energy is not large for the energy of the lowest $\pi K$ scattering state, the single exponential behavior for the time correlation function is seen only for a very large time region. In this case, it is very difficult to extract the scattering state with the small statistical error. In order to overcome this possible problem, we use the variational method [24] with two kinds of operators, $\Omega_{0}^{(1 / 2)}$ and $\Omega_{1}^{(1 / 2)}$ $\left(\bar{\Omega}_{0}^{(1 / 2)}\right.$ and $\left.\bar{\Omega}_{1}^{(1 / 2)}\right)$,

$$
\begin{aligned}
\Omega_{0}^{(1 / 2)}\left(t_{1}, t\right) & =\frac{1}{\sqrt{3}}\left(K^{+}\left(t_{1}\right) \pi^{0}(t)-\sqrt{2} K^{0}\left(t_{1}\right) \pi^{+}(t)\right) \cdot \mathrm{e}^{m_{K}\left(t_{1}-t\right)}, \\
\Omega_{1}^{(1 / 2)}(t) & =\bar{s} u(t), \\
\bar{\Omega}_{0}^{(1 / 2)}\left(t_{0}\right) & =\frac{1}{\sqrt{3}}\left(W_{K^{+}}\left(t_{0}+1\right) W_{\pi^{0}}\left(t_{0}\right)-\sqrt{2} W_{K^{0}}\left(t_{0}+1\right) W_{\pi^{+}}\left(t_{0}\right)\right), \\
\bar{\Omega}_{1}^{(1 / 2)}\left(t_{0}\right) & =W_{\bar{s} u}\left(t_{0}+1\right),
\end{aligned}
$$

where $K^{0}(t)$ and $\pi^{0}(t)$ are the local operator for the $K^{0}$ and $\pi^{0}$ meson at the time slice $t$ with the zero spatial momentum, respectively. $W_{K^{0}}(t), W_{\pi^{0}}(t)$, and $W_{\bar{s} u}(t)$ are the wall-source operators for the corresponding mesons at the time slice $t$, respectively. The exponential factor $\mathrm{e}^{m_{K}\left(t_{1}-t\right)}$ in the definition of $\Omega_{0}^{(1 / 2)}\left(t_{1}, t\right)$ is introduced like as for the other channels.

We construct the $2 \times 2$ matrix of a time correlation function,

$$
G_{i j}^{(1 / 2)}(t)=\left\langle 0\left|\Omega_{i}^{(1 / 2)}\left(t_{1}, t\right) \bar{\Omega}_{j}^{(1 / 2) \dagger}\left(t_{0}\right)\right| 0\right\rangle \quad(i, j=0,1) .
$$

In method I the sink operators are set to the equal time slice, $t_{1}=t$, and they simultaneously run over whole time extent. For the $\pi K(I=1 / 2)$ system, we need to repeat solving quark propagators for the whole time extent as explained later and the computational costs become huge. Thus, we only employ method II for this channel. For $t_{0} \ll t \ll t_{1}$, $G_{i j}^{(1 / 2)}(t)$ can be written by

$$
G_{i j}^{(1 / 2)}(t)=\sum_{n} w_{i n} v_{n j} \cdot \mathrm{e}^{-E_{n}\left(t-t_{0}\right)},
$$

where

$$
\begin{aligned}
w_{0 n} & =\frac{1}{\sqrt{3}}\left[\left\langle 0\left|K^{+}\right| K\right\rangle\left\langle K\left|\pi^{0}\right| E_{n}\right\rangle-\sqrt{2}\left\langle 0\left|K^{0}\right| K\right\rangle\left\langle K\left|\pi^{+}\right| E_{n}\right\rangle\right], \\
w_{1 n} & =\left\langle 0\left|\Omega_{1}^{(1 / 2)}\right| E_{n}\right\rangle, \\
v_{n j} & =\left\langle E_{n}\left|\bar{\Omega}_{j}^{(1 / 2) \dagger}\right| 0\right\rangle .
\end{aligned}
$$

$|K\rangle$ is the kaon state and $\left|E_{n}\right\rangle$ is the $n$th energy eigenstate of the $\pi K$ state with the energy eigenvalue $E_{n}$. We assume that the lowest two states dominate the time correlation function in a large time region. With this assumption, we can extract the energy $E_{n}$ by a single exponential fit for two eigenvalues $\bar{G}_{n}(t)(n=0,1)$ as

$$
\bar{G}_{n}(t)=\operatorname{Ev}\left[\left[G^{(1 / 2)}\left(t_{R}\right)\right]^{-1} \cdot G^{(1 / 2)}(t)\right]_{n}=\mathrm{e}^{-E_{n}\left(t-t_{R}\right)},
$$

where $\operatorname{Ev}[M]_{n}$ means the $n$th eigenvalue of the matrix $M$ and $t_{R}$ is some reference time. The function $\bar{G}_{n}(t)$ is the time correlation function of an optimal operator $\phi_{n}$ for the $n$th energy eigenstate $\left|E_{n}\right\rangle$, whose property is $\left\langle 0\left|\phi_{n}(t)\right| E_{m}\right\rangle=\delta_{n m} \mathrm{e}^{-E_{n} t}$.

Next, we explain the construction of the time correlation functions by quark propagators. The time correlation functions of the $\pi \pi(I=2)$ and $K K(I=1)$ systems are given by 


$$
\begin{aligned}
G^{(2)}(t)= & G_{\operatorname{direct~} 1}^{\pi \pi \rightarrow \pi \pi}(t)+G_{\operatorname{direct} 2}^{\pi \pi \rightarrow \pi \pi}(t)-G_{\operatorname{cross} 1}^{\pi \pi \rightarrow \pi \pi}(t) \\
& -G_{\operatorname{cross} 2}^{\pi \pi \rightarrow \pi \pi}(t), \\
G^{(1)}(t)= & G_{\text {direct 1 }}^{K \rightarrow K K}(t)+G_{\text {direct2 }}^{K K \rightarrow K K}(t)-G_{\operatorname{cross} 1}^{K K \rightarrow K K}(t) \\
& -G_{\operatorname{cross} 2}^{K K \rightarrow K K}(t),
\end{aligned}
$$

$$
\begin{aligned}
G_{\text {direct 1 }}^{\pi \pi \rightarrow \pi \pi}(t) & =\left\langle X^{\pi}\left(t_{1} \mid t_{0}+1\right) X^{\pi}\left(t \mid t_{0}\right) \cdot \mathrm{e}^{m_{\pi} \cdot\left(t_{1}-t\right)}\right\rangle, \\
G_{\text {direct } 2}^{\pi \pi \rightarrow \pi \pi}(t) & =\left\langle X^{\pi}\left(t \mid t_{0}+1\right) X^{\pi}\left(t_{1} \mid t_{0}\right) \cdot \mathrm{e}^{m_{\pi} \cdot\left(t_{1}-t\right)}\right\rangle, \\
G_{\text {cross } 1}^{\pi \pi \rightarrow \pi}(t) & =\left\langle\operatorname{Tr}\left[U^{l l}\left(t_{1} \mid t_{0}\right) U^{l l \dagger}\left(t \mid t_{0}\right)\right] \cdot \mathrm{e}^{m_{\pi} \cdot\left(t_{1}-t\right)}\right\rangle, \\
G_{\text {cross 2 }}^{\pi \pi \rightarrow \pi \pi}(t) & =\left\langle\operatorname{Tr}\left[U^{l l}\left(t \mid t_{0}\right) U^{l l \dagger}\left(t_{1} \mid t_{0}\right)\right] \cdot \mathrm{e}^{m_{\pi} \cdot\left(t_{1}-t\right)}\right\rangle, \\
G_{\text {direct 1 }}^{K K \rightarrow K K}(t) & =\left\langle X^{K}\left(t_{1} \mid t_{0}+1\right) X^{K}\left(t \mid t_{0}\right) \cdot \mathrm{e}^{m_{K} \cdot\left(t_{1}-t\right)}\right\rangle, \\
G_{\text {direct 2 }}^{K K \rightarrow K K}(t) & =\left\langle X^{K}\left(t \mid t_{0}+1\right) X^{K}\left(t_{1} \mid t_{0}\right) \cdot \mathrm{e}^{m_{K} \cdot\left(t_{1}-t\right)}\right\rangle, \\
G_{\text {cross } 1}^{K K K}(t) & =\left\langle\operatorname{Tr}\left[U^{s l}\left(t_{1} \mid t_{0}\right) U^{l s^{\dagger}}\left(t \mid t_{0}\right)\right] \cdot \mathrm{e}^{m_{K} \cdot\left(t_{1}-t\right)}\right\rangle, \\
G_{\text {cross 2 }}^{K K \rightarrow K K}(t) & =\left\langle\operatorname{Tr}\left[U^{s l}\left(t \mid t_{0}\right) U^{l s^{\dagger}}\left(t_{1} \mid t_{0}\right)\right] \cdot \mathrm{e}^{m_{K} \cdot\left(t_{1}-t\right)}\right\rangle .
\end{aligned}
$$

In Eq. (20) the angle bracket refers to the expectation value over the gauge configurations, and the trace is taken for the color and spinor indices. The exponential factors $\mathrm{e}^{m_{\pi}\left(t_{1}-t\right)}$

where
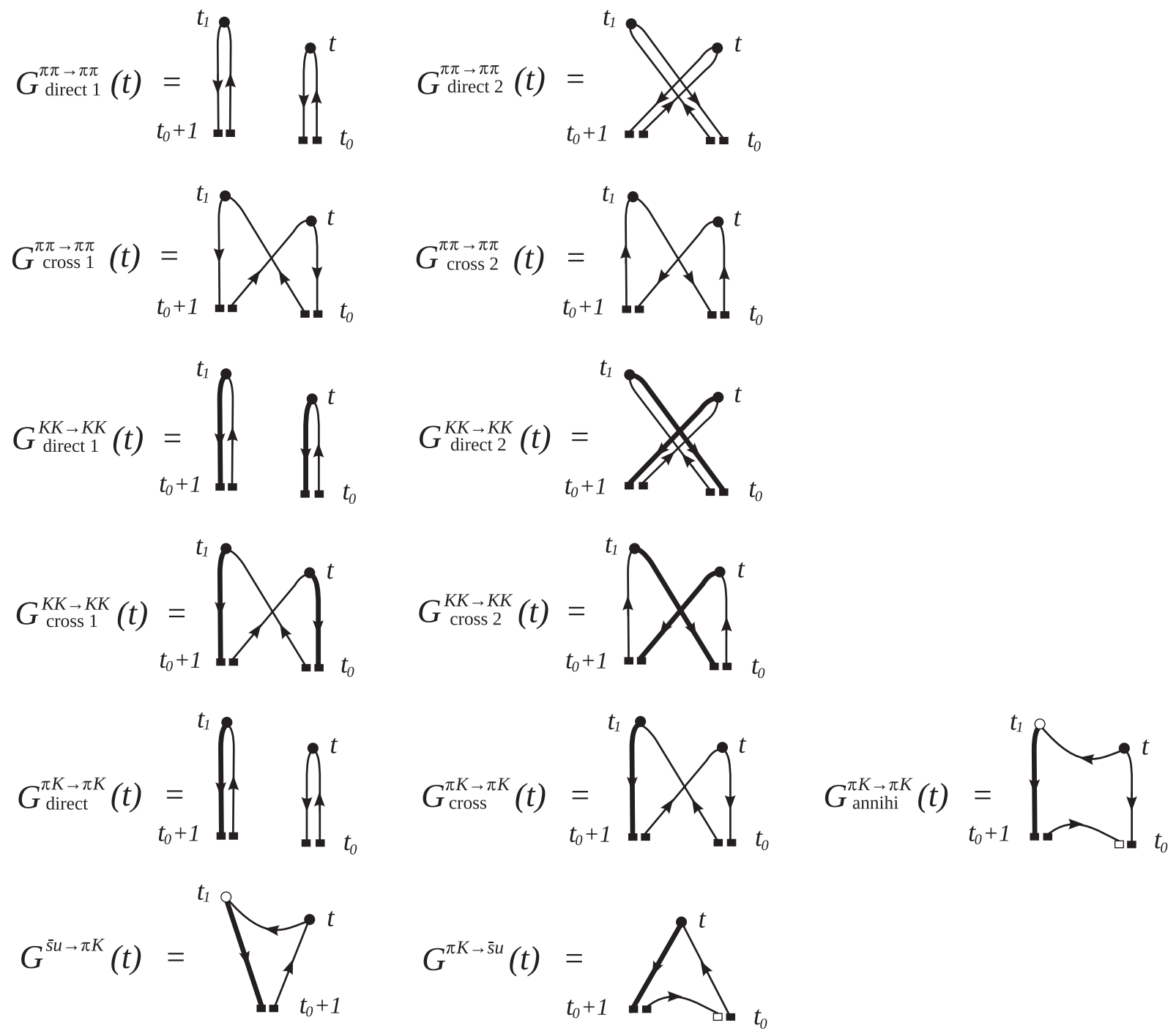

FIG. 1. List of the diagrams employed to calculate the time correlation function for the $S$-wave $\pi \pi(I=2), K K(I=1), \pi K(I=3 / 2)$, and $\pi K(I=1 / 2)$ systems. The thin (thick) lines represent the up/down (strange) quark propagators. The time runs upward. The circles are the local operators for the $\pi, K$, and $\bar{s} u$ mesons at the time slice specified in each diagram, and the squares are the wall-source operators for these mesons. The open symbols mean the operators summed over $\mathbf{y}$ in Eqs. (31) and (32). 
and $\mathrm{e}^{m_{K}\left(t_{1}-t\right)}$ come from the definitions of $\Omega^{(2)}$ in Eq. (6) and $\Omega^{(1)}$ in Eq. (10), respectively. The indices $l$ and $s$ mean the up/down and strange quark, respectively. $X^{\pi}\left(t \mid t_{s}\right)$, $X^{K}\left(t \mid t_{s}\right)$, and $U^{f_{1} f_{2}}\left(t \mid t_{s}\right)$ are defined by

$$
\begin{aligned}
X^{\pi}\left(t \mid t_{s}\right)= & \sum_{\mathbf{x}} \operatorname{Tr}\left[Q^{l \dagger}\left(\mathbf{x}, t \mid t_{s}\right) Q^{l}\left(\mathbf{x}, t \mid t_{s}\right)\right], \\
X^{K}\left(t \mid t_{s}\right)= & \sum_{\mathbf{x}} \operatorname{Tr}\left[Q^{s \dagger}\left(\mathbf{x}, t \mid t_{s}\right) Q^{l}\left(\mathbf{x}, t \mid t_{s}\right)\right], \\
U_{A B}^{f_{1} f_{2}}\left(t \mid t_{s}\right)= & \sum_{\mathbf{x}} \sum_{C} Q_{C A}^{f_{1} *}\left(\mathbf{x}, t \mid t_{s}+1\right) \\
& \times Q_{C B}^{f_{2}}\left(\mathbf{x}, t \mid t_{s}\right)\left(f_{1}, f_{2}=l, s\right),
\end{aligned}
$$

with the quark propagator with the wall source

$$
Q_{A B}^{f}\left(\mathbf{x}, t \mid t_{s}\right)=\sum_{\mathbf{y}}\left(D^{-1}\right)_{A B}^{f}\left(\mathbf{x}, t ; \mathbf{y}, t_{s}\right) \quad(f=l, s),
$$

where $A, B$, and $C$ refer to color and spinor indices.

The quark diagrams for the components in Eq. (20) are shown in Fig. 1. The thin (thick) lines represent the up/ down (strange) quark propagators. The time runs upward in the diagrams. The circles are the local operators for the $\pi$,
$K$, and $\bar{s} u$ mesons at the time slice specified in each diagram, and the squares are the wall-source operators for these mesons.

$G^{(3 / 2)}(t)$ and $G_{i j}^{(1 / 2)}(t)$ are constructed by

$$
G^{(3 / 2)}(t)=G_{\text {direct }}^{\pi K \rightarrow \pi K}(t)-G_{\text {cross }}^{\pi K \rightarrow \pi K}(t),
$$

$G_{00}^{(1 / 2)}(t)=G_{\text {direct }}^{\pi K \rightarrow \pi K}(t)+\frac{1}{2} G_{\text {cross }}^{\pi K \rightarrow \pi K}(t)-\frac{3}{2} G_{\text {annihi }}^{\pi K \rightarrow \pi K}(t)$,

$$
G_{01}^{(1 / 2)}(t)=-\sqrt{\frac{3}{2}} G^{\bar{s} u \rightarrow \pi K}(t),
$$

$$
G_{10}^{(1 / 2)}(t)=-\sqrt{\frac{3}{2}} G^{\pi K \rightarrow \bar{s} u}(t)
$$

$$
G_{11}^{(1 / 2)}(t)=G^{\bar{s} u}\left(t \mid t_{0}+1\right)
$$

where

$$
\begin{aligned}
G_{\text {direct }}^{\pi K \rightarrow \pi K}(t) & =\left\langle X^{K}\left(t_{1} \mid t_{0}+1\right) X^{\pi}\left(t \mid t_{0}\right) \cdot \mathrm{e}^{m_{K} \cdot\left(t_{1}-t\right)}\right\rangle, \\
G_{\text {cross }}^{\pi K \rightarrow \pi K}(t) & =\left\langle\operatorname{Tr}\left[U^{s l}\left(t_{1} \mid t_{0}\right) U^{l l \dagger}\left(t \mid t_{0}\right)\right] \cdot \mathrm{e}^{m_{K} \cdot\left(t_{1}-t\right)}\right\rangle, \\
G_{\text {annihi }}^{\pi K \rightarrow \pi K}(t) & =\left\langle\sum_{\mathbf{x}} \operatorname{Tr}\left[W^{\dagger}\left(\mathbf{x}, t\left|t_{1}\right| t_{0}+1\right) V\left(\mathbf{x}, t \mid t_{0}+1\right)\right] \cdot \mathrm{e}^{m_{K} \cdot\left(t_{1}-t\right)}\right\rangle, \\
G^{\bar{s} u \rightarrow \pi K}(t) & =\left\langle\sum_{\mathbf{x}} \operatorname{Tr}\left[W^{\dagger}\left(\mathbf{x}, t\left|t_{1}\right| t_{0}+1\right) \gamma_{5} Q^{l}\left(\mathbf{x}, t \mid t_{0}+1\right)\right] \cdot \mathrm{e}^{m_{K} \cdot\left(t_{1}-t\right)}\right\rangle, \\
G^{\pi K \rightarrow \bar{s} u}(t) & =\left\langle\sum_{\mathbf{x}} \operatorname{Tr}\left[Q^{s \dagger}\left(\mathbf{x}, t \mid t_{0}+1\right) \gamma_{5} V\left(\mathbf{x}, t \mid t_{0}+1\right)\right]\right\rangle, \\
G^{\bar{s} u}\left(t \mid t_{s}\right) & =\left\langle\sum_{\mathbf{x}} \operatorname{Tr}\left[\gamma_{5} Q^{s \dagger}\left(\mathbf{x}, t \mid t_{s}\right) \gamma_{5} Q^{l}\left(\mathbf{x}, t \mid t_{s}\right)\right]\right\rangle .
\end{aligned}
$$

In Eq. (30) the exponential factor $\mathrm{e}^{m_{K}\left(t_{1}-t\right)}$ comes from the definitions of $\Omega^{(3 / 2)}$ in Eq. (12) and $\Omega_{0}^{(1 / 2)}$ in Eq. (13). $V\left(\mathbf{x}, t \mid t_{s}\right)$ and $W\left(\mathbf{x}, t\left|t_{a}\right| t_{s}\right)$ are defined by

$$
\begin{aligned}
V_{A B}\left(\mathbf{x}, t \mid t_{s}\right)= & \sum_{C} Q_{A C}^{l}\left(\mathbf{x}, t \mid t_{s}-1\right) \\
& \times\left\{\gamma_{5} \sum_{\mathbf{y}} Q^{l}\left(\mathbf{y}, t_{s}-1 \mid t_{s}\right)\right\}_{C B},
\end{aligned}
$$

$$
\begin{aligned}
W_{A B}\left(\mathbf{x}, t\left|t_{a}\right| t_{s}\right)= & \sum_{\mathbf{y}} \sum_{C}\left(D^{-1}\right)_{A C}^{l}\left(\mathbf{x}, t ; \mathbf{y}, t_{a}\right) \\
& \times\left[\gamma_{5} Q^{s}\left(\mathbf{y}, t_{a} \mid t_{s}\right)\right]_{C B},
\end{aligned}
$$

where the square bracket in Eq. (32) is taken as the source in solving the propagator. The quark diagram for the components in Eq. (30) are plotted in Fig. 1. The open symbols mean the operators summed over $\mathbf{y}$ in Eqs. (31) and (32). In method I, we must solve $W\left(\mathbf{x}, t|t| t_{0}+1\right)$ for 
each $t$ in the calculation of $G_{\text {annihi }}^{\pi K \rightarrow \pi K}(t)$ and $G^{\bar{s} u \rightarrow \pi K}(t)$. On the other hand, in method II, we need to solve it only once at $t_{1}$. Therefore, the computational cost is reduced for the $\pi K(I=1 / 2)$ system.

We solve four kinds of $Q$-type propagators in Eq. (24) with $\left(f, t_{s}\right)=\left(l, t_{0}+1\right),\left(s, t_{0}+1\right),\left(l, t_{0}\right),\left(s, t_{0}\right)$. We also solve one $W$-type propagator in Eq. (32) with $\left(t_{a}, t_{s}\right)=\left(t_{1}, t_{0}+1\right)$. Thus, we solve five quark propagators for each configuration.

\section{Simulation parameters}

The calculations are carried out with $N_{f}=2+1$ full QCD configurations generated by the PACS-CS Collaboration [25] using the Iwasaki gauge action at $\beta=1.90$ and nonperturbatively $\mathcal{O}(a)$-improved Wilson quark action with $C_{S W}=1.715$ on a $32^{3} \times 64$ lattice. The lattice cutoff is $a^{-1}=2.194(10) \mathrm{GeV} \quad[a=$ $0.08995(40) \mathrm{fm}]$ determined from the $\Omega$-baryon mass. The spatial extent of the lattice is $L a=2.878(13) \mathrm{fm}$ [26]. The statistical error of $a$ is not included in the following analysis. The quark-mass parameters, the corresponding hadron masses, and the number of configurations are listed in Table I.

The quark propagators are calculated with the same action as the configuration generation. They are solved on the configurations at every 20 trajectories for $\kappa_{u d}=0.13781$, and 10 trajectories for the others. The Dirichlet boundary condition is imposed for the temporal direction and the periodic boundary condition for the spatial directions. The wall source is used with the gauge configurations fixed to the Coulomb gauge. The time slices of the source operators are $t_{0}=12$ and $t_{0}+1=13$, and the time slice of the fixed sink operator is set at $t_{1}=53$. We adopt $t_{R}=18$ as the reference time for the diagonalization for the $\pi K(I=1 / 2)$ system. The statistical errors are evaluated by the jackknife analysis with a bin size of $110 \mathrm{MD}$ time for $\kappa_{u d}=0.13781$, and $125 \mathrm{MD}$ time for the others. Here, the MD time is the number of trajectories multiplied by the trajectory length $\tau$, which takes $\tau=0.25$ for $\kappa_{u d}=0.13781$ and 0.13770 , and $\tau=0.5$ for the other $\kappa_{u d}$.

We calculate the time correlation functions on the gauge configurations shifted by $T_{\text {shift }}$ in the temporal direction and take an average of them to improve the statistics. We use $T_{\text {shift }}$ listed in Table I, but do not include $T_{\text {shift }}=0$ for the analysis of the $K K(I=1)$ system in all the quark masses.

\section{RESULTS OF THE SCATTERING LENGTH}

\section{A. Time correlation functions and effective masses}

The time correlation functions of the $\pi \pi(I=2)$, $K K(I=1), \quad \pi K(I=3 / 2)$, and $\pi K(I=1 / 2)$ systems which are defined in Eqs. (5), (9), (11), and (14), are plotted in the columns (a), (b), (c), and (d) of Fig. 2, respectively. Each row in Fig. 2 represents the time correlation functions for $m_{\pi}=0.17,0.30,0.41,0.57$ and $0.71 \mathrm{GeV}$. For the repulsive channels, $\pi \pi(I=2), K K(I=$ $1)$ and $\pi K(I=3 / 2)$, we employ both methods I and II for the calculation of the time correlation function as explained before. The two results are compared in the figure. For $\pi K(I=1 / 2)$ plotted in column (d), the absolute values of each component of $\left|G^{(1 / 2)}(t)\right|$ are presented. As discussed in the previous section, we employ only method II for this channel. The open symbols represent the diagonal elements of $G^{(1 / 2)}(t)$. The filled symbols represent the off-diagonal elements, whose signs are reversed.

The effective masses for the repulsive channel, $\pi \pi(I=2), K K(I=1)$, and $\pi K(I=3 / 2)$ systems are plotted in columns (a), (b), and (c) of Fig. 3, respectively. Each row of Fig. 3 represents the effective masses for $m_{\pi}=0.17,0.30,0.41,0.57$, and $0.71 \mathrm{GeV}$. We show the results with method I by squares and method II by circles. We observe clear plateaus for all the cases. We also find that the two methods give the same results of the effective masses. This supports that our choice of $t_{1}=53$ is enough large for the dominance of the one particle state in the time correlation functions.

For the $\pi K(I=1 / 2)$ system, we calculate the effective masses for the eigenvalues $\bar{G}_{n}(t)=$ $\operatorname{EV}\left[\left[G^{(1 / 2)}\left(t_{R}\right)\right]^{-1} \cdot G^{(1 / 2)}(t)\right]_{n}$ in Eq. (17) for the lowest $(n=0)$ state and the next-lowest $(n=1)$ state. They are plotted in columns (a) and (b) of Fig. 4. One sees that the effective mass of the $n=0$ state shows a clear plateau in the small $m_{\pi}$ region $\left(m_{\pi}=0.17-0.57 \mathrm{GeV}\right)$, while it does only a short plateau at the large $m_{\pi}\left(m_{\pi}=0.71 \mathrm{GeV}\right)$. The reason will be discussed in Sec. III C.

TABLE I. The quark-mass parameters and corresponding hadron masses. We do not include $T_{\text {shift }}=0$ for the analysis of the $K K(I=1)$ system.

\begin{tabular}{lccccc}
\hline \hline$\kappa_{u d}$ & $\kappa_{s}$ & $m_{\pi}[\mathrm{GeV}]$ & $m_{K}[\mathrm{GeV}]$ & $N_{\text {conf }}$ & $T_{\text {shift }}$ \\
\hline 0.13781 & 0.13640 & $0.1661(58)$ & $0.5594(23)$ & 154 & $0,16,32,48$ \\
0.13770 & 0.13640 & $0.2973(23)$ & $0.5975(17)$ & 800 & $0,16,32$ \\
0.13754 & 0.13640 & $0.4144(16)$ & $0.6401(13)$ & 450 & $0,16,32,48$ \\
0.13727 & 0.13640 & $0.5746(13)$ & $0.7190(12)$ & 400 & $0,16,32,48$ \\
0.13700 & 0.13640 & $0.7069(12)$ & $0.7953(12)$ & 400 & $0,16,32,48$ \\
\hline \hline
\end{tabular}


(a) $\pi \pi(l=2)$

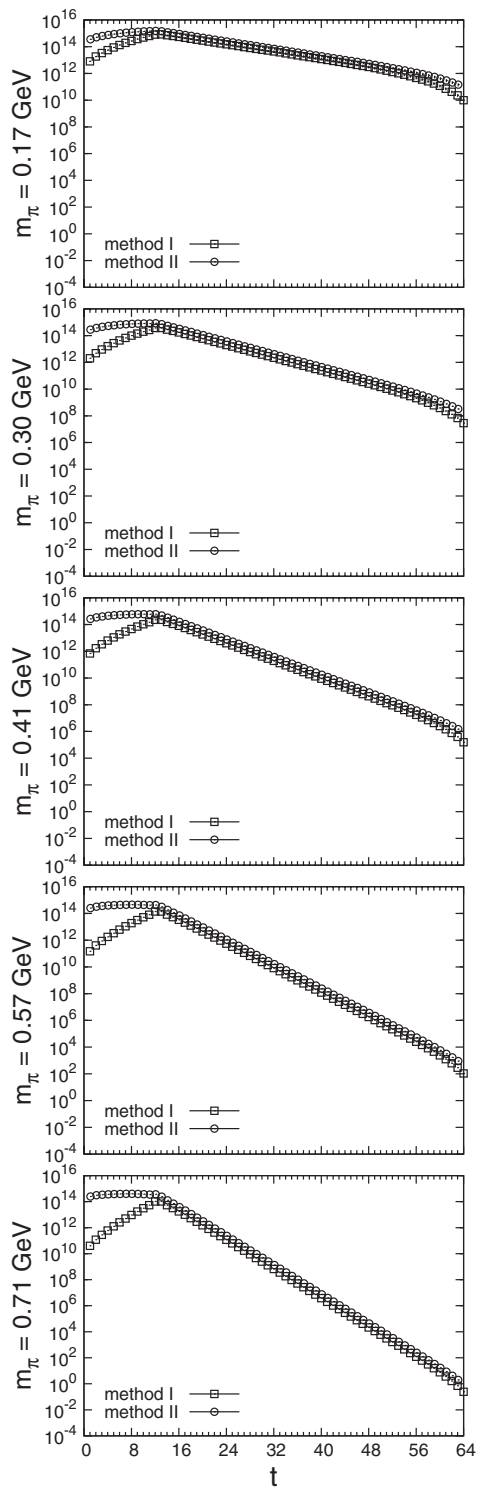

(b) $\mathrm{KK}(\mathrm{l}=1)$

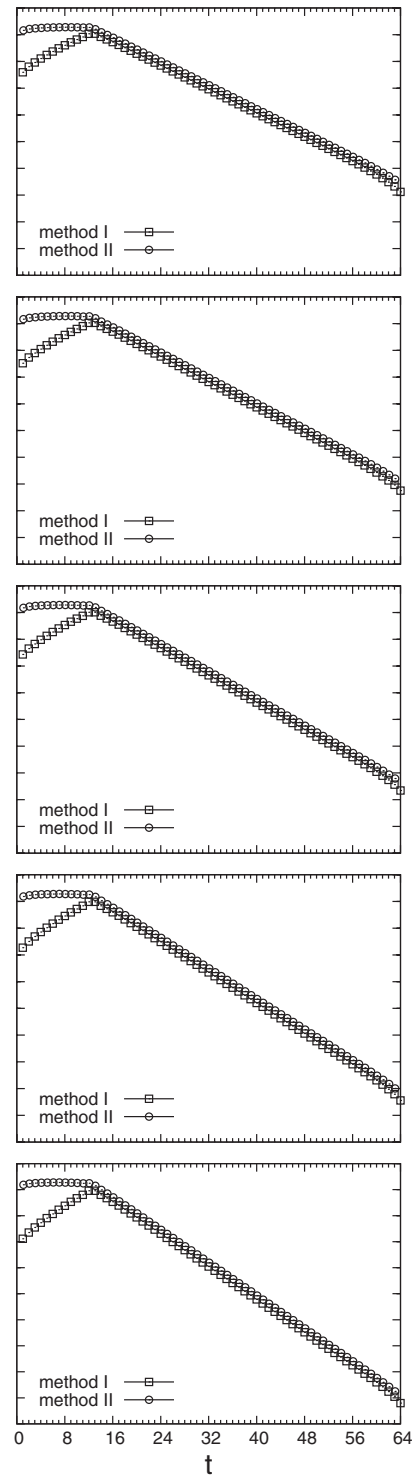

(c) $\pi \mathrm{K}(\mathrm{I}=3 / 2)$

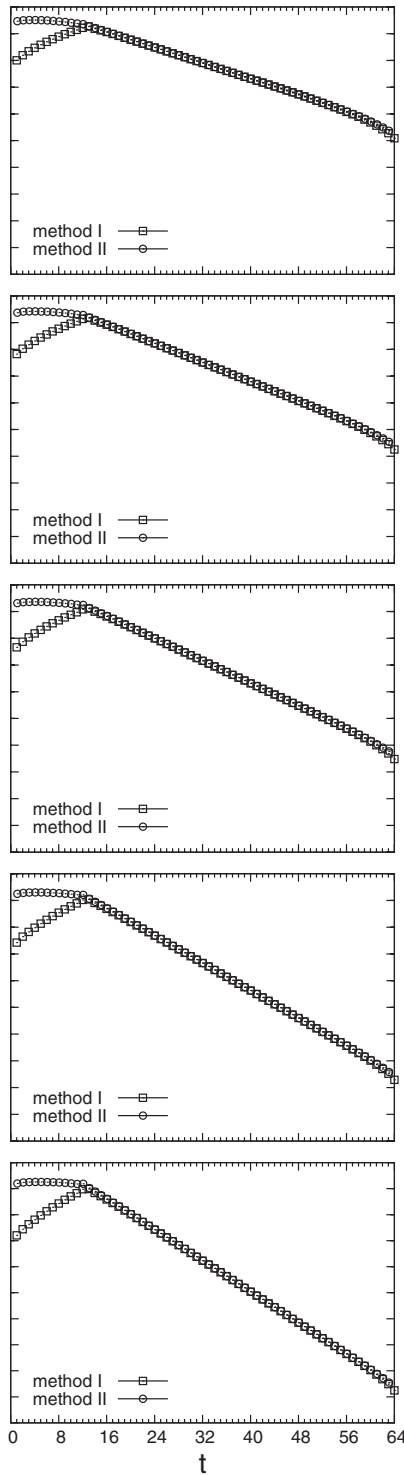

(d) $\pi \mathrm{K}(\mathrm{l}=1 / 2)$

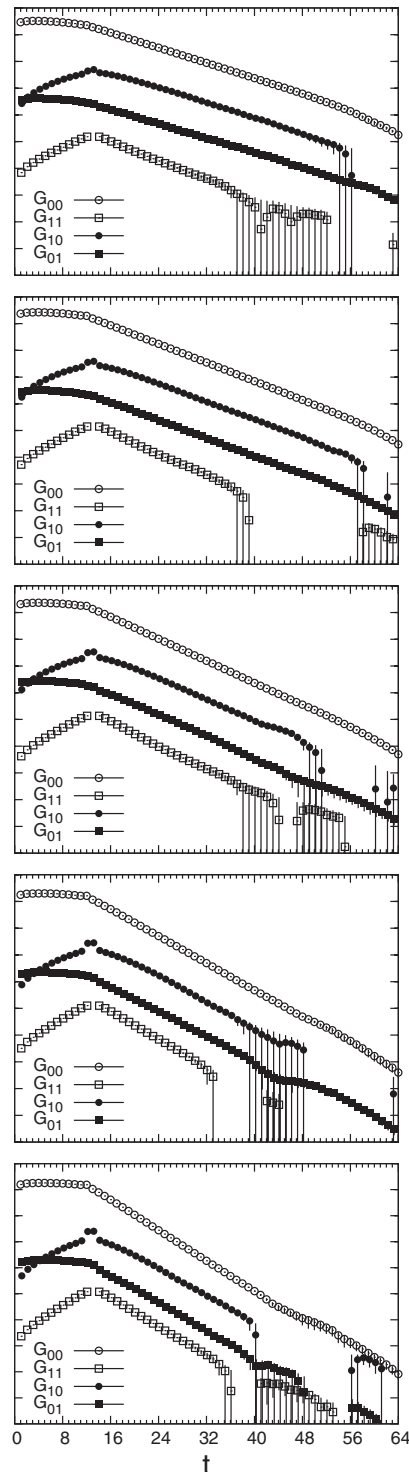

FIG. 2. The time correlation functions for (a) the $\pi \pi(I=2)$, (b) the $K K(I=1)$, (c) the $\pi K(I=3 / 2)$, and (d) the $\pi K(I=1 / 2)$ systems at $m_{\pi}=0.17,0.30,0.41,0.57$, and $0.71 \mathrm{GeV}$. In columns (a), (b), and (c), the results of the two methods, methods I (squares) and II (circles), are shown. In column (d), the open symbols represent the diagonal elements of $G^{(1 / 2)}(t)$. The filled symbols represent the off-diagonal elements, whose signs are reversed.

\section{B. Scattering length for repulsive channels $[\pi \pi(I=2), K K(I=1)$ and $\pi K(I=3 / 2)]$}

For the $\pi \pi(I=2), K K(I=1)$, and $\pi K(I=3 / 2)$ systems, we extract the energy of the lowest state by a single exponential fit for the time correlation functions, $G^{(2)}(t)$, $G^{(1)}(t)$, and $G^{(3 / 2)}(t)$ in Eqs. (5), (9), and (11). As shown in Sec. III A, the effective masses of the time correlation functions obtained by methods I and II give the consistent results. Thus, we average over the energies extracted from these two time correlation functions. In Tables II, III, and IV, we tabulate the fit range, the energy $E$, and the scattering momentum $k$. We evaluate the scattering phase shift $\delta_{0}(k)$ by substituting $k$ into Eq. (1), which is also tabulated in the tables.
In all the cases, $\tan \delta_{0}$ is negative, so that the interaction is repulsive. If the interaction between two particles is not strong, then the scattering momentum $\left|k^{2}\right|$ takes a small value and $\tan \delta_{0}(k) / k$ can be expanded in terms of $k^{2}$ as

$$
\left[\tan \delta_{0}(k) / k\right]^{-1}=\frac{1}{a_{0}}+\frac{1}{2} r_{\text {eff }} k^{2}+\mathcal{O}\left(k^{4}\right),
$$

where $a_{0}$ is the scattering length and $r_{\text {eff }}$ is the effective range. In the following, we assume that $\mathcal{O}\left(k^{2}\right)$ and the higher terms can be neglected in (33) at all $m_{\pi}$ for the repulsive channel, and we regard the first term of (33) as the inverse of the scattering length. 
(a) $\pi \pi(l=2)$
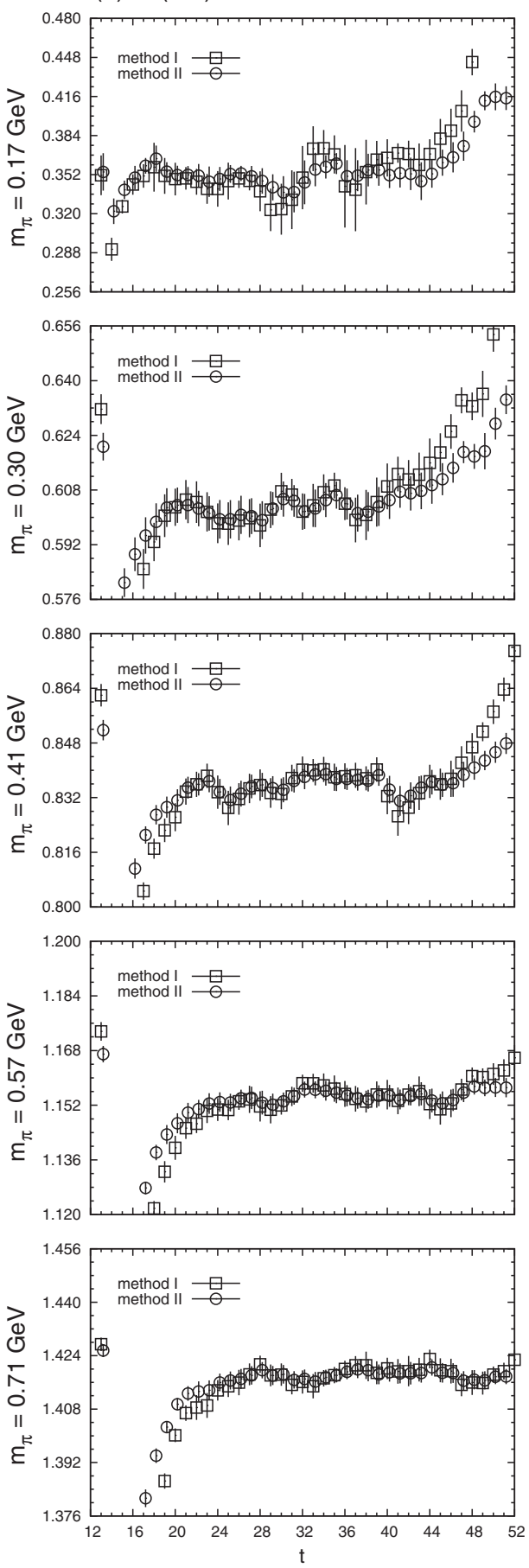

(b) $\mathrm{KK}(\mathrm{l}=1)$

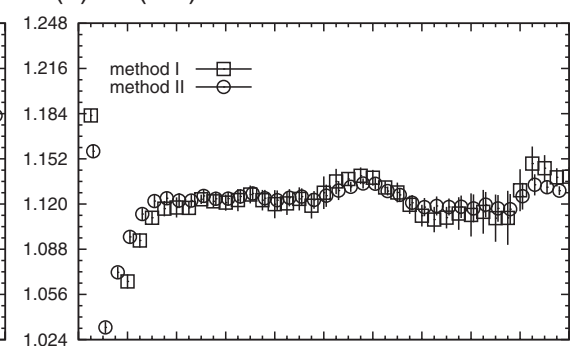

(c) $\pi \mathrm{K}(\mathrm{l}=3 / 2)$
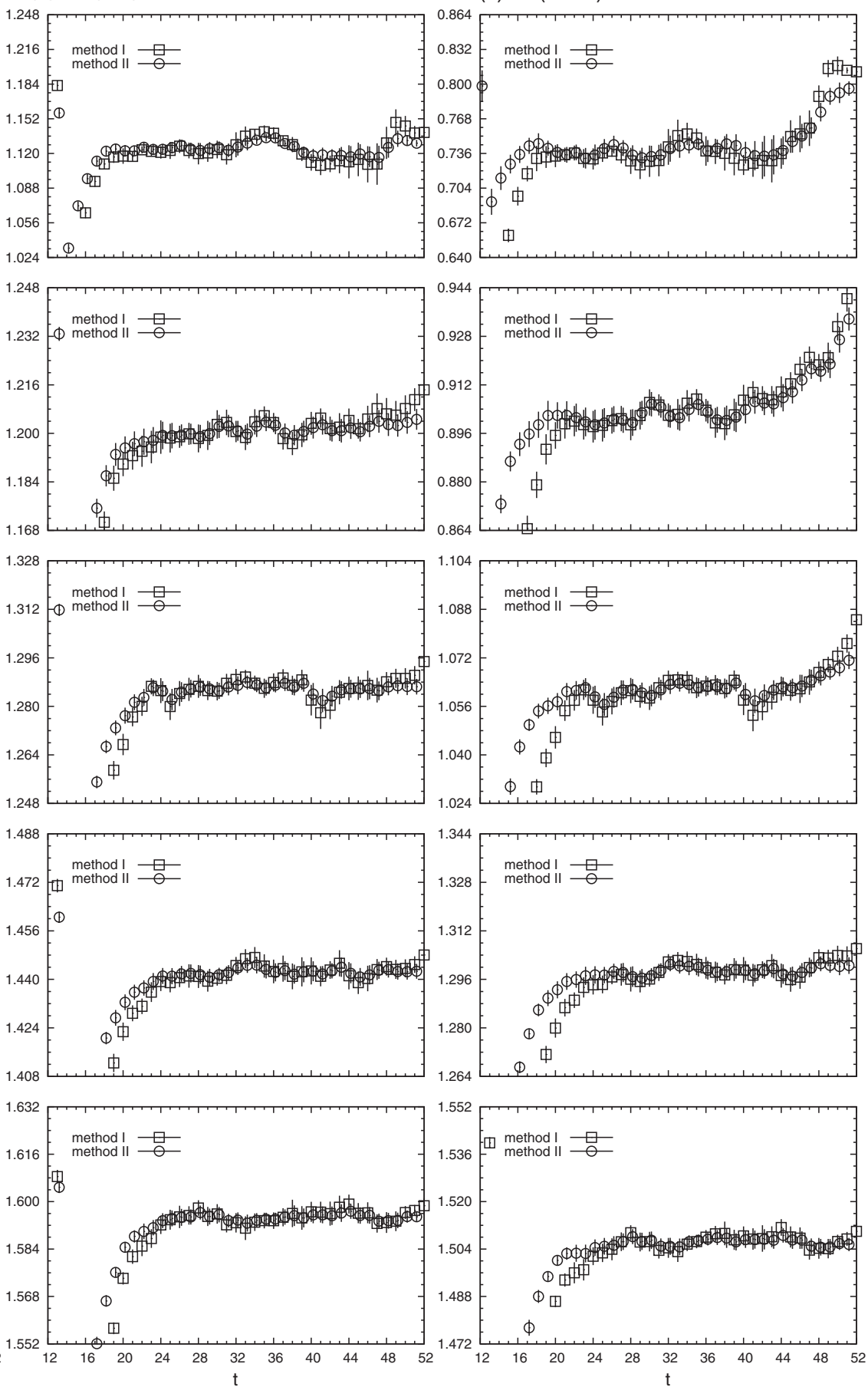

FIG. 3. The effective masses in the physical unit $(\mathrm{GeV})$ for (a) the $\pi \pi(I=2)$, (b) the $K K(I=1)$, and (c) the $\pi K(I=3 / 2)$ systems at $m_{\pi}=0.17,0.30,0.41,0.57$, and $0.71 \mathrm{GeV}$. The results of the two methods, methods I (squares) and II (circles), are shown.

\section{Scattering length for the attractive channel $[\pi K(I=1 / 2)]$}

In order to clearly show the contamination from the higher states for the $\pi K(I=1 / 2)$ system, we consider the ratios,

$$
\begin{aligned}
R_{i}(t) \equiv & \frac{G_{i i}^{(1 / 2)}(t)}{G_{i i}^{(1 / 2)}\left(t_{R}\right)} \cdot\left[\mathrm{e}^{-\left(m_{\pi}+m_{K}\right)\left(t-t_{R}\right)}\right]^{-1} \quad(i=0,1), \\
D_{n}(t) \equiv & \operatorname{EV}\left[\left[G^{(1 / 2)}\left(t_{R}\right)\right]^{-1} \cdot G^{(1 / 2)}(t)\right]_{n} \\
& \cdot\left[\mathrm{e}^{-\left(m_{\pi}+m_{K}\right)\left(t-t_{R}\right)}\right]^{-1} \quad(n=0,1) .
\end{aligned}
$$


(a) $\pi \mathrm{K}(\mathrm{I}=1 / 2)$ lowest
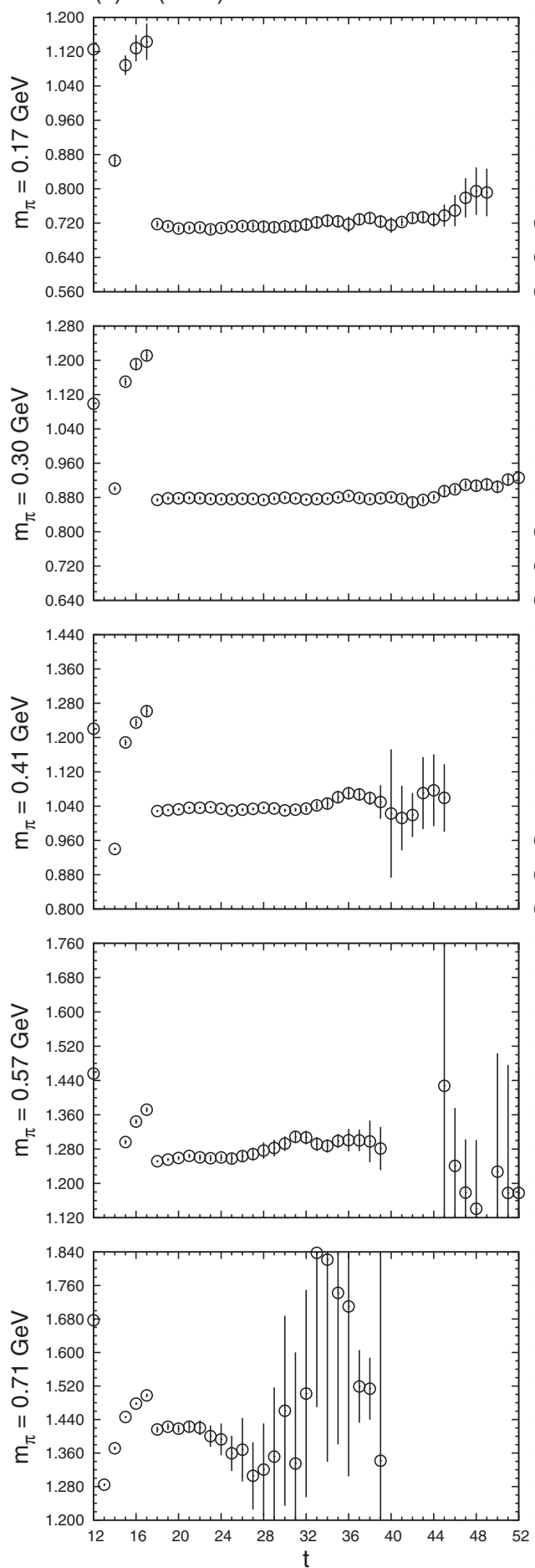

(b) $\pi \mathrm{K}(\mathrm{I}=1 / 2)$ next
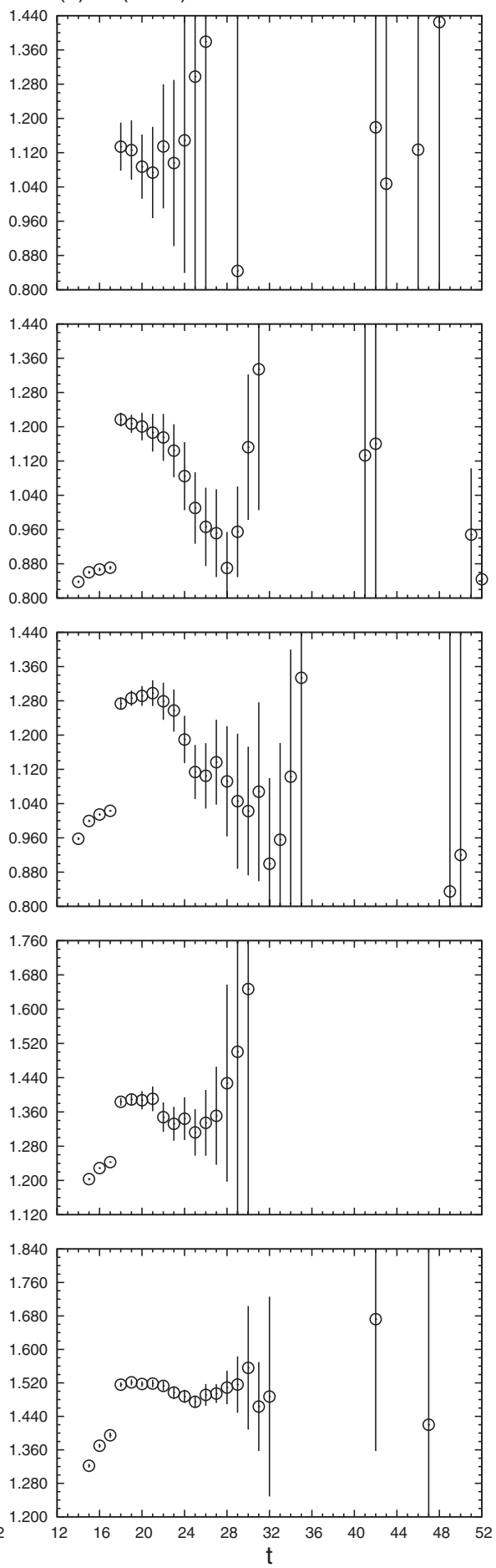

FIG. 4. The effective masses in the physical unit $(\mathrm{GeV})$ for (a) the lowest $\pi K(I=1 / 2)(n=0)$ and (b) the next-lowest $\pi K(I=1 / 2)$ $(n=1)$ systems at $m_{\pi}=0.17,0.30,0.41,0.57$ and $0.71 \mathrm{GeV}$.

In Fig. $5, R_{0}(t)$ (open circles), $R_{1}(t)$ (open squares), $D_{0}(t)$ (filled circles), and $D_{1}(t)$ (filled squares) are plotted. Each row of Fig. 5 represents the results for $m_{\pi}=0.17,0.30$, $0.41,0.57$ and $0.71 \mathrm{GeV}$, respectively. Note that for $m_{\pi}=0.71 \mathrm{GeV}, R_{1}(t)$ is plotted in the left panel and
$R_{0}(t)$ in the right panel, which is different from the other masses.

We find that the difference between $R_{0}(t)$ and $D_{0}(t)$ is small for $m_{\pi}=0.17-0.30 \mathrm{GeV}$ in Fig. 5. This means that the $\pi K$-type operator $\left[\Omega_{0}^{(1 / 2)}\right]$ has a large overlap with the 


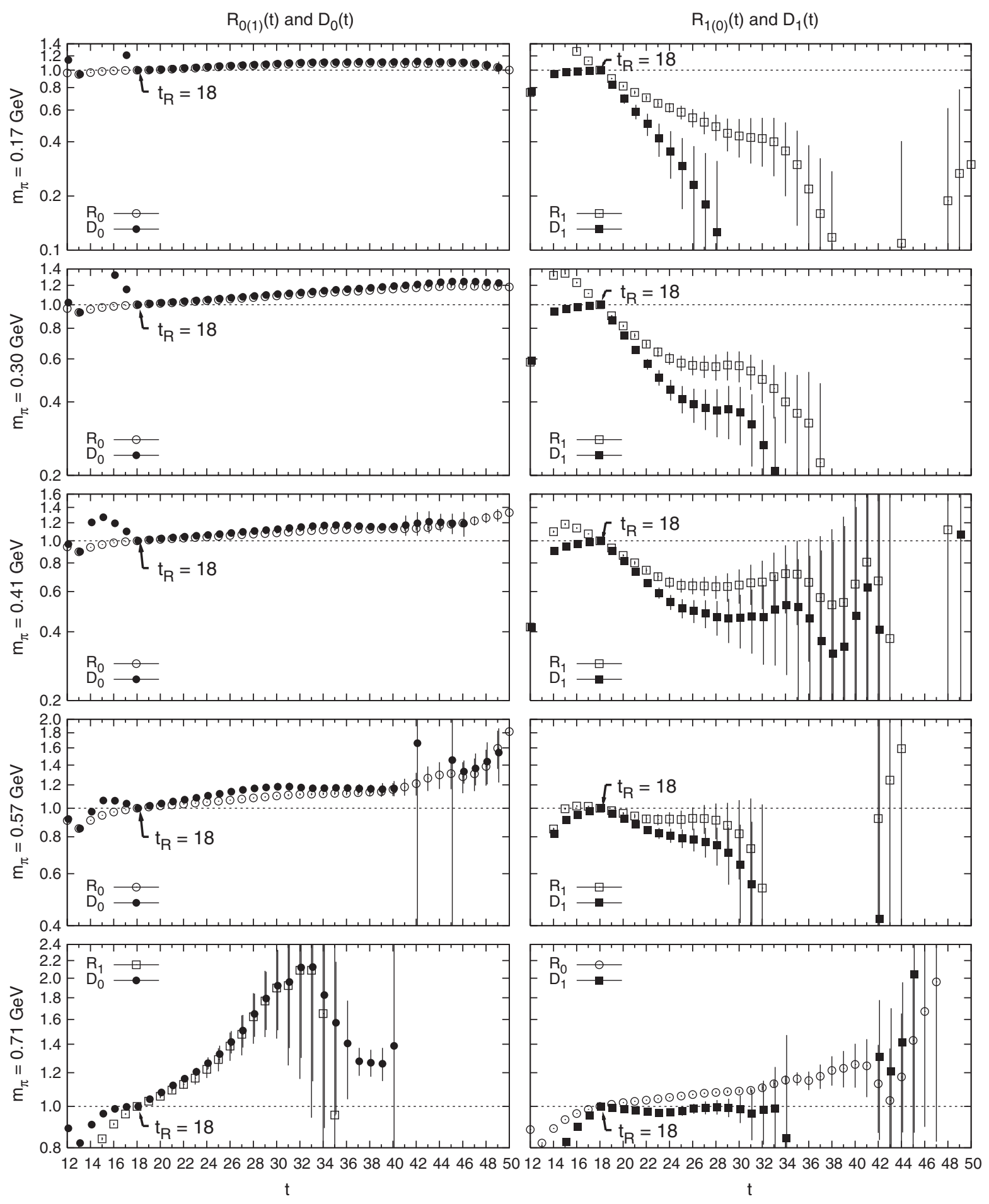

FIG. 5. $\quad R_{0}(t)$ (open circles), $R_{1}(t)$ (open squares), $D_{0}(t)$ (filled circles), and $D_{1}(t)$ (filled squares) are plotted. Each row represents the results at $m_{\pi}=0.17,0.30,0.41,0.57$, and $0.71 \mathrm{GeV}$, respectively.

lowest $(n=0)$ state for the small quark mass. On the other hand, at $m_{\pi}=0.71 \mathrm{GeV}, D_{0}(t)$ is very different from $R_{0}(t)$ and is similar to $R_{1}(t)$. This means that the operator which has a large overlap with the $n=0$ state is the $\bar{s} u$-type operator $\left[\Omega_{1}^{(1 / 2)}\right]$ for the large quark mass. We can also read out this tendency from the effective masses.
As we have observed in Fig. 4 , at $m_{\pi}=0.71 \mathrm{GeV}$, the statistical error of the effective mass of the $n=0$ state is larger than that of the next-lowest $(n=1)$ state. This can be attributed to a fact that $G_{11}^{(1 / 2)}(t)$ has a larger statistical error, and is the dominant contribution to the $n=0$ state. 
TABLE II. The energy $E$, the scattering momentum $k$, and the scattering phase shift $\delta_{0}(k)$ for the lowest state of the $S$-wave $\pi \pi(I=2)$ system. The fit range for the extraction of the energy $E$ from the time correlation are also tabulated. $E^{\text {free }}=2 m_{\pi}$ is also shown for a guide.

\begin{tabular}{llccccc}
\hline \hline$\kappa_{u d}$ & & 0.13781 & 0.13770 & 0.13754 & 0.13727 & 0.13700 \\
$m_{\pi}$ & {$[\mathrm{GeV}]$} & $0.1661(58)$ & $0.2973(23)$ & $0.4144(16)$ & $0.5746(13)$ & $0.7069(12)$ \\
\hline Fit range & & $22-42$ & $23-43$ & $24-44$ & $25-46$ & $25-46$ \\
$E^{\text {free }}$ & {$[\mathrm{GeV}]$} & $0.332(12)$ & $0.5947(47)$ & $0.8288(32)$ & $1.1492(26)$ & $1.4137(25)$ \\
$E$ & {$[\mathrm{GeV}]$} & $0.347(10)$ & $0.6024(49)$ & $0.8357(33)$ & $1.1543(27)$ & $1.4180(25)$ \\
$k^{2}$ & {$\left[\mathrm{GeV}^{2}\right]$} & $0.00256(25)$ & $0.00229(23)$ & $0.00285(18)$ & $0.00292(18)$ & $0.00302(11)$ \\
{$\left[\tan \delta_{0}(k) / k\right]^{-1}$} & {$[\mathrm{GeV}]$} & $-1.78(16)$ & $-1.96(18)$ & $-1.611(89)$ & $-1.574(87)$ & $-1.531(48)$ \\
{$\left[\tan \delta_{0}(k) / k\right]$} & {$[\mathrm{fm}]$} & $-0.1113(97)$ & $-0.1010(92)$ & $-0.1225(68)$ & $-0.1254(70)$ & $-0.1289(41)$ \\
{$\left[\tan \delta_{0}(k) / k\right] \cdot m_{\pi}$} & & $-0.0936(64)$ & $-0.152(14)$ & $-0.257(14)$ & $-0.365(21)$ & $-0.462(15)$ \\
$\delta_{0}(k)$ & {$[\mathrm{deg}]$} & $-1.64(22)$ & $-1.40(20)$ & $-1.90(16)$ & $-1.97(17)$ & $-2.05(10)$ \\
\hline \hline
\end{tabular}

TABLE III. The same as Table II for the $S$-wave $K K(I=1)$ system $\left(E^{\text {free }}=2 m_{K}\right)$.

\begin{tabular}{|c|c|c|c|c|c|c|}
\hline $\begin{array}{l}\kappa_{u d} \\
m_{\pi} \\
\end{array}$ & {$[\mathrm{GeV}]$} & $\begin{array}{c}0.13781 \\
0.1661(58)\end{array}$ & $\begin{array}{c}0.13770 \\
0.2973(23)\end{array}$ & $\begin{array}{c}0.13754 \\
0.4144(16)\end{array}$ & $\begin{array}{c}0.13727 \\
0.5746(13)\end{array}$ & $\begin{array}{c}0.13700 \\
0.7069(12)\end{array}$ \\
\hline Fit range & & $22-42$ & $23-43$ & $24-44$ & $25-46$ & $25-46$ \\
\hline$E^{\text {free }}$ & {$[\mathrm{GeV}]$} & $1.1188(46)$ & $1.1950(35)$ & $1.2802(26)$ & $1.4381(24)$ & $1.5905(24)$ \\
\hline$E$ & {$[\mathrm{GeV}]$} & $1.1256(43)$ & $1.2003(35)$ & $1.2858(25)$ & $1.4423(25)$ & $1.5944(24)$ \\
\hline$k^{2}$ & {$\left[\mathrm{GeV}^{2}\right]$} & $0.00382(71)$ & $0.00321(20)$ & $0.00362(22)$ & $0.00303(32)$ & $0.00307(22)$ \\
\hline$\left[\tan \delta_{0}(k) / k\right]^{-1}$ & {$[\mathrm{GeV}]$} & $-1.26(20)$ & $-1.450(80)$ & $-1.306(70)$ & $-1.53(14)$ & $-1.510(94)$ \\
\hline$\left[\tan \delta_{0}(k) / k\right]$ & {$[\mathrm{fm}]$} & $-0.158(25)$ & $-0.1361(75)$ & $-0.1511(80)$ & $-0.130(12)$ & $-0.1308(81)$ \\
\hline$\left[\tan \delta_{0}(k) / k\right] \cdot m_{K}$ & & $-0.448(71)$ & $-0.412(23)$ & $-0.490(26)$ & $-0.471(44)$ & $-0.527(33)$ \\
\hline$\left[\tan \delta_{0}(k) / k\right] \cdot m_{\pi}$ & & $-0.133(19)$ & $-0.205(11)$ & $-0.317(17)$ & $-0.377(35)$ & $-0.468(29)$ \\
\hline$\delta_{0}(k)$ & {$[\mathrm{deg}]$} & $-2.84(71)$ & $-2.24(19)$ & $-2.64(22)$ & $-2.07(30)$ & $-2.10(21)$ \\
\hline
\end{tabular}

We show the fit range, the energy $E$, and the scattering momentum $k$ in Table $\mathrm{V}$ for the lowest state $(n=0)$ and Table VI for the next-lowest state $(n=1)$. We find that $k^{2}$ is negative and the interaction is attractive for the $n=0$ state. We evaluate the scattering phase shift $\delta_{0}(k)$ by substituting $k$ into Eq. (1), which are also tabulated in the tables. For the $n=1$ state at $m_{\pi}=0.17$ and $0.71 \mathrm{GeV}, k^{2}$ gets across the divergence points of the function $\sqrt{4 \pi} g_{00}(k ; 1)$ within the statistical errors and the values of $\left[\tan \delta_{0}(k) / k\right]^{-1}$ diverge. For the $n=1$ state at $m_{\pi}=0.30$ and $0.41 \mathrm{GeV}$, $k^{2}$ gets across the zero points of $\sqrt{4 \pi} g_{00}(k ; 1)$ within the statistical errors and the values of $\tan \delta_{0}(k) / k$ diverge. In Table VI, these values are omitted.

In Fig. 6, the energy eigenvalues of the $n=0$ and $n=1$ states are plotted as a function of $m_{\pi}^{2}$. The two dashed lines are the energies of the $n=0$ and $n=1$ states for the noninteracting $\pi K$ system, which are given by

$$
\begin{gathered}
E_{0}^{\mathrm{free}}=m_{\pi}+m_{K}, \\
E_{1}^{\mathrm{free}}=\sqrt{m_{\pi}^{2}+(2 \pi / L a)^{2}}+\sqrt{m_{K}^{2}+(2 \pi / L a)^{2}} .
\end{gathered}
$$

TABLE IV. The same as Table II for the $S$-wave $\pi K(I=3 / 2)$ system $\left(E^{\text {free }}=m_{\pi}+m_{K}\right) \cdot \mu_{\pi K} \equiv m_{\pi} m_{K} /\left(m_{\pi}+m_{K}\right)$ is the reduced mass of $\pi$ and $K$.

\begin{tabular}{llccccc}
\hline \hline$\kappa_{u d}$ & & 0.13781 & 0.13770 & 0.13754 & 0.13727 & 0.13700 \\
$m_{\pi}$ & {$[\mathrm{GeV}]$} & $0.1661(58)$ & $0.2973(23)$ & $0.4144(16)$ & $0.5746(13)$ & $0.7069(12)$ \\
\hline Fit range & & $22-42$ & $23-43$ & $24-44$ & $25-46$ & $25-46$ \\
$E^{\text {free }}$ & {$[\mathrm{GeV}]$} & $0.7261(82)$ & $0.8949(38)$ & $1.0545(28)$ & $1.2937(25)$ & $1.5021(24)$ \\
$E$ & {$[\mathrm{GeV}]$} & $0.7371(73)$ & $0.9019(39)$ & $1.0609(29)$ & $1.2985(26)$ & $1.5062(25)$ \\
$k^{2}$ & {$\left[\mathrm{GeV}^{2}\right]$} & $0.00302(39)$ & $0.00281(14)$ & $0.00320(19)$ & $0.00307(20)$ & $0.00308(13)$ \\
{$\left[\tan \delta_{0}(k) / k\right]^{-1}$} & {$[\mathrm{GeV}]$} & $-1.54(18)$ & $-1.629(70)$ & $-1.453(78)$ & $-1.509(86)$ & $-1.503(54)$ \\
{$\left[\tan \delta_{0}(k) / k\right]$} & {$[\mathrm{fm}]$} & $-0.129(15)$ & $-0.1212(52)$ & $-0.1359(72)$ & $-0.1309(75)$ & $-0.1313(47)$ \\
{$\left[\tan \delta_{0}(k) / k\right] \cdot \mu_{\pi K}$} & & $-0.0838(92)$ & $-0.1219(54)$ & $-0.1733(93)$ & $-0.212(12)$ & $-0.2491(91)$ \\
{$\left[\tan \delta_{0}(k) / k\right] \cdot m_{\pi}$} & & $-0.108(12)$ & $-0.1826(82)$ & $-0.285(15)$ & $-0.381(22)$ & $-0.471(17)$ \\
$\delta_{0}(k)$ & {$[\mathrm{deg}]$} & $-2.06(36)$ & $-1.87(12)$ & $-2.23(19)$ & $-2.10(19)$ & $-2.12(12)$ \\
\hline \hline
\end{tabular}


TABLE V. The same as Table II for the lowest $(n=0)$ state of the $S$-wave $\pi K(I=1 / 2)$ system $\left(E_{0}^{\text {free }}=m_{\pi}+m_{K}\right)$ except for $\sigma_{0}(k)$. $\sigma_{0}(k)$ is a real function defined by the analytic continuation as $\tan \sigma_{0} / \kappa=\tan \delta_{0}(k) / k\left(\kappa^{2} \equiv-k^{2}\right)$. It is noted that $\sigma_{0}(k)$ is not the physical scattering phase shift.

\begin{tabular}{|c|c|c|c|c|c|c|}
\hline $\begin{array}{l}\kappa_{u d} \\
m_{\pi}\end{array}$ & {$[\mathrm{GeV}]$} & $\begin{array}{c}0.13781 \\
0.1661(58)\end{array}$ & $\begin{array}{c}0.13770 \\
0.2973(23)\end{array}$ & $\begin{array}{c}0.13754 \\
0.4144(16)\end{array}$ & $\begin{array}{c}0.13727 \\
0.5746(13)\end{array}$ & $\begin{array}{c}0.13700 \\
0.7069(12)\end{array}$ \\
\hline fit range & & $20-42$ & $20-40$ & $20-32$ & $20-27$ & $20-27$ \\
\hline$E_{0}^{\text {free }}$ & {$[\mathrm{GeV}]$} & $0.7261(82)$ & $0.8949(38)$ & $1.0545(28)$ & $1.2937(25)$ & $1.5021(24)$ \\
\hline$E$ & {$[\mathrm{GeV}]$} & $0.7126(84)$ & $0.8772(41)$ & $1.0340(27)$ & $1.2605(57)$ & $1.413(15)$ \\
\hline$k^{2}$ & {$\left[\mathrm{GeV}^{2}\right]$} & $-0.00323(59)$ & $-0.00689(49)$ & $-0.01018(78)$ & $-0.0209(29)$ & $-0.064(10)$ \\
\hline$\left[\tan \delta_{0}(k) / k\right]^{-1}$ & {$[\mathrm{GeV}]$} & $1.07(23)$ & $0.380(42)$ & $0.184(32)$ & $-0.038(32)$ & $-0.239(26)$ \\
\hline$\left[\tan \delta_{0}(k) / k\right]$ & {$[\mathrm{fm}]$} & $0.188(41)$ & $0.520(59)$ & $1.08(18)$ & $-5.9(6.8)$ & $-0.826(89)$ \\
\hline$\left[\tan \delta_{0}(k) / k\right] \cdot \mu_{\pi K}$ & & $0.122(27)$ & $0.523(60)$ & $1.37(24)$ & $-10 .(11)$. & $-1.57(17)$ \\
\hline$\left[\tan \delta_{0}(k) / k\right] \cdot m_{\pi}$ & & $0.158(36)$ & $0.784(89)$ & $2.26(39)$ & $-17 .(20)$. & $-2.96(32)$ \\
\hline$\sigma_{0}(k)$ & [deg] & $3.12(98)$ & $12.3(1.8)$ & $28.8(5.1)$ & $-75 .(11)$. & $-46.68(74)$ \\
\hline
\end{tabular}

TABLE VI. The same as Table II for the next-lowest $(n=1)$ state of the $S$-wave $\pi K(I=1 / 2)$ system. We take $E_{1}^{\text {free }}=\sqrt{m_{\pi}^{2}+p^{2}}+$ $\sqrt{m_{K}^{2}+p^{2}}$ with $p=2 \pi / L a$.

\begin{tabular}{lcccccc}
\hline \hline$\kappa_{u d}$ & & 0.13781 & 0.13770 & 0.13754 & 0.13727 & 0.13700 \\
$m_{\pi}$ & {$[\mathrm{GeV}]$} & $0.1661(58)$ & $0.2973(23)$ & $0.4144(16)$ & $0.5746(13)$ & $0.7069(12)$ \\
\hline Fit range & & $20-42$ & $20-40$ & $20-32$ & $20-27$ & $20-27$ \\
$E_{1}^{\text {free }}$ & {$[\mathrm{GeV}]$} & $1.1679(40)$ & $1.2600(26)$ & $1.3693(21)$ & $1.5563(21)$ & $1.7322(21)$ \\
$E$ & {$[\mathrm{GeV}]$} & $1.16(18)$ & $1.139(67)$ & $1.246(42)$ & $1.366(30)$ & $1.507(10)$ \\
$k^{2}$ & {$\left[\mathrm{GeV}^{2}\right]$} & $0.182(99)$ & $0.116(37)$ & $0.106(26)$ & $0.047(20)$ & $0.0036(78)$ \\
{$\left[\tan \delta_{0}(k) / k\right]-1$} & {$[\mathrm{GeV}]$} & $\ldots$ & $0.18(29)$ & $0.10(15)$ & $-0.174(93)$ & $\ldots$ \\
{$\left[\tan \delta_{0}(k) / k\right]$} & {$[\mathrm{fm}]$} & $\ldots$ & $\ldots$ & $\ldots$ & $-1.18(63)$ & $\ldots$ \\
{$\left[\tan \delta_{0}(k) / k\right] \cdot \mu_{\pi K}$} & & $\ldots$ & $\ldots$ & $\ldots$ & $-1.9(1.0)$ & $-3.4(1.8)$ \\
{$\left[\tan \delta_{0}(k) / k\right] \cdot m_{\pi}$} & & $\ldots$ & $\ldots$ & $\ldots$ & $-52 .(20)$ \\
$\delta_{0}(k)$ & {$[\mathrm{deg}]$} & $\ldots$ & $\ldots$ & $\ldots$ \\
\hline \hline
\end{tabular}

The continuous values of these for $m_{\pi}^{2}$ are given by a linear interpolation from the measured value of $m_{K}^{2}$. From Fig. 6, we find that the energies of the $n=0$ states lie near $E_{0}^{\text {free }}$ in the small $m_{\pi}$ region $\left(m_{\pi}=0.17-0.41 \mathrm{GeV}\right)$, while it is lower than $E_{0}^{\text {free }}$ in the largest $m_{\pi}\left(m_{\pi}=0.71 \mathrm{GeV}\right)$. The

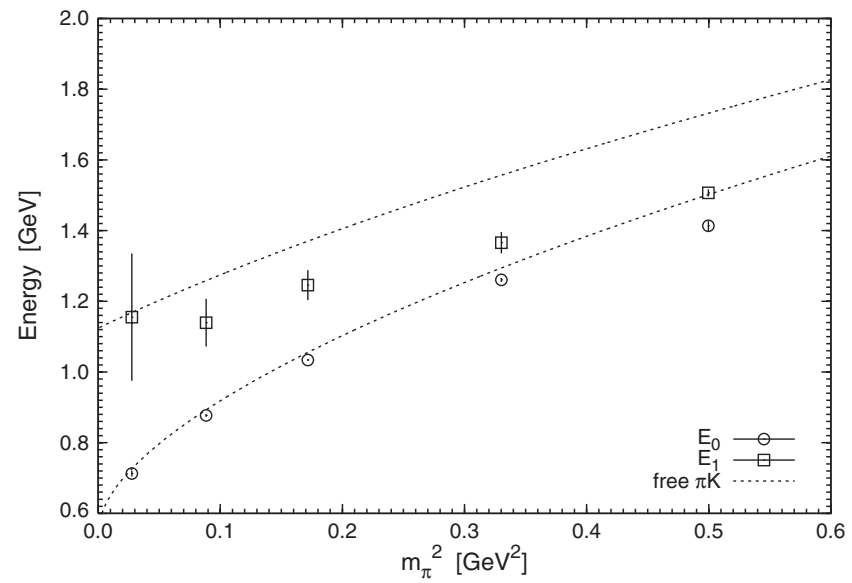

FIG. 6. The energies of the lowest state (circles) and the nextlowest state (squares) for the $\pi K(I=1 / 2)$ system as a function of $m_{\pi}^{2}$. The energies of the free $\pi K$ system are also shown by dashed lines. energy of the $n=1$ state lies near $E_{1}^{\text {free }}$ in the smallest $m_{\pi}$ $\left(m_{\pi}=0.17 \mathrm{GeV}\right)$. It deviates from $E_{1}^{\text {free }}$, and gets closer to $E_{0}^{\text {free }}$ for larger $m_{\pi}$. We note that similar features of the $n=0$ state in the scalar meson channel have already reported in Refs. [27-30].

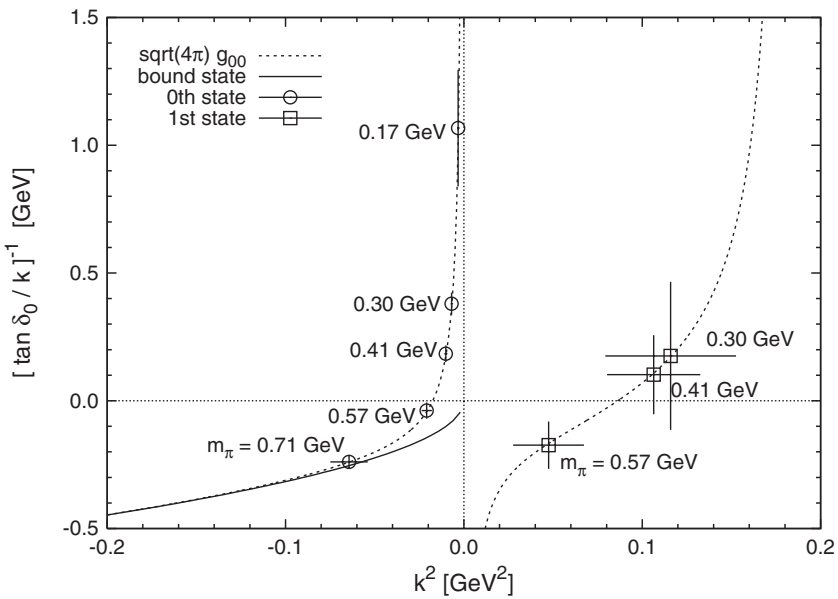

FIG. 7. $\sqrt{4 \pi} g_{00}(k ; 1)$ (dashed line), and the results of $\left[\tan \delta_{0}(k) / k\right]^{-1}$ (open symbols) as a function of $k^{2}$. A solid line is $\tan \delta_{0}(k)=-i$ which corresponds to a formation of the bound state. 
TABLE VII. The quark formulation, the number of flavor $N_{f}$, the lattice spacing $a$, the spatial extent $L a$, and the pion-mass range of the present and previous studies.

\begin{tabular}{|c|c|c|c|c|c|}
\hline & Quark formulation & $N_{f}$ & $a[\mathrm{fm}]$ & $L a[\mathrm{fm}]$ & $m_{\pi}[\mathrm{GeV}]$ \\
\hline The present work & Improved Wilson & $2+1$ & 0.090 & 2.9 & $0.17-0.71$ \\
\hline NPLQCD [12,15,18] & Improved staggered (sea) + domain-wall (valence) & $2+1$ & 0.125 & 2.5 & $0.29-0.60$ \\
\hline $\mathrm{RBC}$ and UK [3] & Domain-wall & $2+1$ & 0.114 & 1.8 & $0.43-0.67$ \\
\hline \multirow[t]{3}{*}{ ETM [13] } & Maximally twisted-mass & 2 & 0.067 & 2.1 & 0.31 \\
\hline & & & 0.086 & 2.1 & $0.39-0.49$ \\
\hline & & & 0.086 & 2.7 & $0.27-0.31$ \\
\hline Yagi et al. [14] & Overlap & 2 & 0.118 & 1.9 & $0.29-0.75$ \\
\hline \multirow[t]{4}{*}{$\mathrm{Fu}[5]$} & Improved staggered & $2+1$ & 0.12 & 2.7 & 0.24 \\
\hline & & & & 2.4 & $0.32-0.37$ \\
\hline & & & 0.15 & 3.0 & 0.24 \\
\hline & & & & 2.5 & $0.33-0.46$ \\
\hline $\mathrm{Fu}[19]$ & Improved staggered & $2+1$ & 0.15 & 2.5 & $0.33-0.46$ \\
\hline Lang et al. [20] & Improved Wilson & 2 & 0.124 & 2.0 & 0.27 \\
\hline
\end{tabular}

In order to more clearly show this phenomena, we plot $\left[\tan \delta_{0}(k) / k\right]^{-1}$ in Fig. 7, where the dashed line is the function given by the right-hand side of Eq. (1). A solid line is $\tan \delta_{0}(k)=-i$. The $n=1$ states at $m_{\pi}=0.17,0.71 \mathrm{GeV}$ are not plotted because they are across the divergent points of $\sqrt{4 \pi} g_{00}(k ; 1)$. Because of the strong attraction, $\left[\tan \delta_{0}(k) / k\right]^{-1}$ of the $n=0$ state changes the sign, and $\tan \delta_{0}(k) \simeq-i$ at $m_{\pi}=0.71 \mathrm{GeV}$. This suggests a bound state formation at $m_{\pi}=0.71 \mathrm{GeV}$.

In the following discussion, we concentrate only on the $n=0$ state, because the statistics of the phase shift for the $n=1$ state is not enough to analyze the quark-mass dependence and obtain the value at the physical quark mass. Figures 6 and 7 suggest that the values of the scattering phase shift of the $n=0$ state for $m_{\pi} \geq$ $0.57 \mathrm{GeV}$ might be strongly affected by the existence of the bound state. In that case, we need the higher order term of $k^{2}$ in Eq. (33) to obtain the scattering length. In the present work, we assume that $\mathcal{O}\left(k^{2}\right)$ terms in Eq. (33) can be neglected for $m_{\pi} \leq 0.41 \mathrm{GeV}$, and we regard $\tan \delta_{0}(k) / k$ as the scattering length $a_{0}$.

\section{Comparison with the previous studies at several quark masses}

In this section we compare our results with the previous studies [3,5,12-15,18-20]. In Table VII, the quark formulation, the number of flavor $N_{f}$, the lattice spacing $a$, the spatial extent $L a$, and the pion mass $m_{\pi}$ for the present and previous studies are summarized. In Fig. $8, a_{0}^{(2)} m_{\pi}$ for $\pi \pi(I=2), \quad a_{0}^{(1)} m_{K}$ for $K K(I=1), a_{0}^{(3 / 2)} \mu_{\pi K}$ for $\pi K(I=3 / 2)$, and $a_{0}^{(1 / 2)} \mu_{\pi K}$ for $\pi K(I=1 / 2)$ are compared for $m_{\pi}<0.63 \mathrm{GeV}$, where $\mu_{\pi K} \equiv m_{\pi} m_{K} /\left(m_{\pi}+\right.$ $\left.m_{K}\right)$ is the reduced mass of $\pi$ and $K$. These calculations
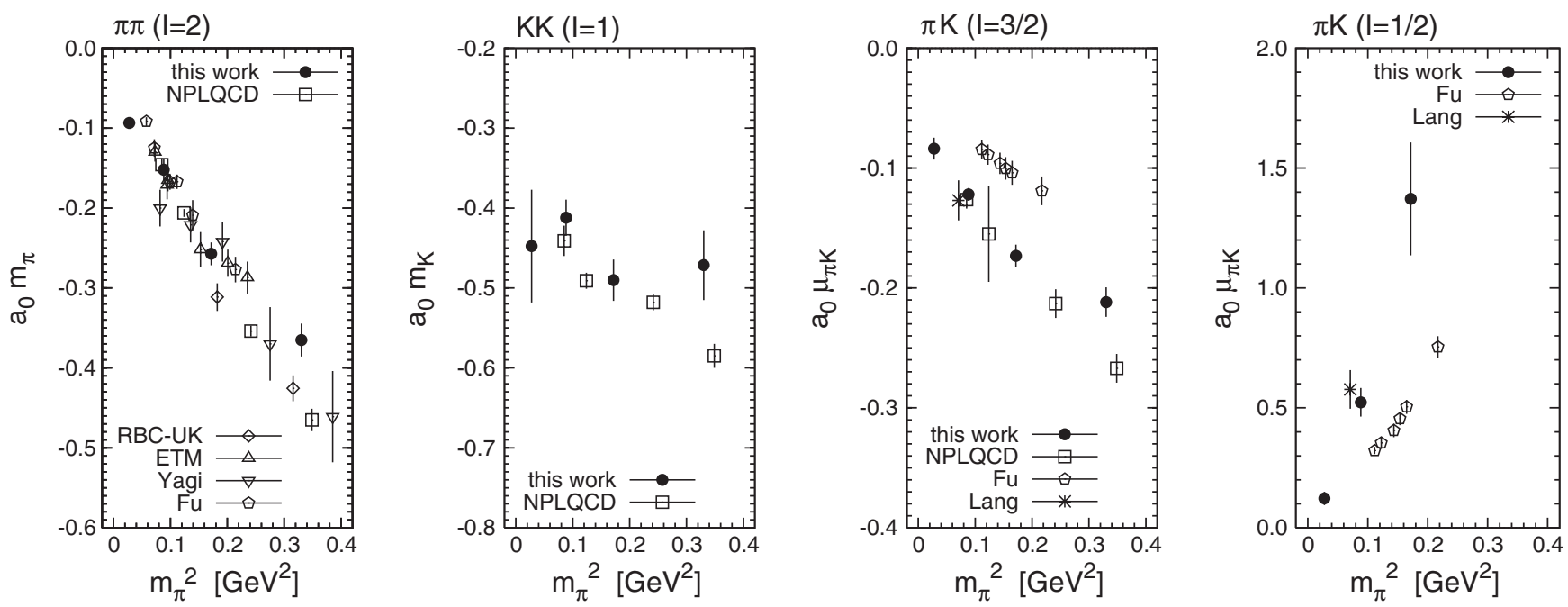

FIG. 8. Comparison of the present results with the previous lattice studies $[3,4,12-15,18-20] . a_{0}^{(2)} m_{\pi}$ for $\pi \pi(I=2), a_{0}^{(1)} m_{K}$ for $K K(I=1), a_{0}^{(3 / 2)} \mu_{\pi K}$ for $\pi K(I=3 / 2)$ and $a_{0}^{(1 / 2)} \mu_{\pi K}$ for $\pi K(I=1 / 2)$ are plotted. For Yagi et al. [14], the finite-volume corrected values are plotted. 
are performed with the different lattice spacings and quark formulations. The strange quark mass is set near the physical strange quark mass. In the figure, we find that all the results for $\pi \pi$ and $K K$ systems are almost consistent. Our results for the $\pi K(I=3 / 2,1 / 2)$ systems are reasonably consistent with the ones of the NPLQCD Collaboration [18] and Lang et al. [20], while large discrepancies from Fu's results [19] are found. We can consider some possible reasons for the discrepancies, e.g., the discretization error, but we need further investigation for a conclusion.

\section{CHIRAL ANALYSIS}

\section{A. Chiral analysis with $\mathcal{O}\left(p^{4}\right)$ ChPT}

In this section, we investigate the quark-mass dependence of the scattering lengths to evaluate the value at the physical quark mass. For this purpose, first, we consider the ChPT formulas in $\mathcal{O}\left(p^{4}\right)$ given in Refs. [31-33]. The scattering lengths of the $\pi \pi(I=2), \quad K K(I=1)$, $\pi K(I=3 / 2)$, and $\pi K(I=1 / 2)$ systems can be written by

$a_{0}^{(2)} m_{\pi}=\frac{m_{\pi}^{2}}{16 \pi f_{\pi}^{2}}\left[-1+\frac{16}{f_{\pi}^{2}}\left[m_{\pi}^{2} \cdot L^{\prime}-\frac{m_{\pi}^{2}}{2} \cdot L_{5}+\zeta^{(2)}\right]\right]$,

$$
a_{0}^{(1)} m_{K}=\frac{m_{K}^{2}}{16 \pi f_{K}^{2}}\left[-1+\frac{16}{f_{K}^{2}}\left[m_{K}^{2} \cdot L^{\prime}-\frac{m_{K}^{2}}{2} \cdot L_{5}+\zeta^{(1)}\right]\right]
$$

$$
\begin{aligned}
a_{0}^{(3 / 2)} \mu_{\pi K}= & \frac{\mu_{\pi K}^{2}}{8 \pi f_{\pi} f_{K}}\left[-1+\frac{16}{f_{\pi} f_{K}}\left[m_{\pi} m_{K} \cdot L^{\prime}\right.\right. \\
& \left.\left.-\frac{m_{\pi}^{2}+m_{K}^{2}}{4} \cdot L_{5}+\zeta^{(3 / 2)}\right]\right], \\
a_{0}^{(1 / 2)} \mu_{\pi K}= & \frac{\mu_{\pi K}^{2}}{8 \pi f_{\pi} f_{K}}\left[2+\frac{16}{f_{\pi} f_{K}}\left[m_{\pi} m_{K} \cdot L^{\prime}\right.\right. \\
& \left.\left.+2 \frac{m_{\pi}^{2}+m_{K}^{2}}{4} \cdot L_{5}+\zeta^{(1 / 2)}\right]\right],
\end{aligned}
$$

where the formulas are written by $\mathcal{O}\left(p^{4}\right)$ values of the masses of the Nambu-Goldstone (NG) bosons $\left(m_{\pi}\right.$ and $\left.m_{K}\right)$ and the decay constants $\left(f_{\pi}\right.$ and $\left.f_{K}\right)$, which are not the parameter of ChPT and depend on the quark masses. The normalization of $f_{\pi}=0.092 \mathrm{GeV}$ at the physical point is adopted. The constants $L_{5}$ and

$$
L^{\prime} \equiv 2 L_{1}+2 L_{2}+L_{3}-2 L_{4}-L_{5} / 2+2 L_{6}+L_{8}
$$

are the LECs defined in Ref. [31] at a renormalization scale $\mu$. In the present work, we adopt $\mu=0.770 \mathrm{GeV}$. $\zeta^{(2),(1),(3 / 2),(1 / 2)}$ are known functions with chiral logarithmic terms, which are given in the Appendix.

In the chiral analysis, we fit our results of the scattering length with the ChPT formulas in Eqs. (37)-(40) for all the channels simultaneously, where the values of $m_{\pi}, m_{K}, f_{\pi}$, and $f_{K}$ in the formulas are fixed to the measured values by the lattice calculations at each quark mass. The free parameters in the fitting are the LECs $\left(L_{5}\right.$ and $\left.L^{\prime}\right)$.

For the other fitting procedure, we rewrite the ChPT formulas in terms of the the quark mass and the decay constant at $m_{\mathrm{q}}=0, F$, and fit our results with the formulas, regarding the LECs $\left(L_{5}\right.$ and $\left.L^{\prime}\right)$ and $F$ as a free parameter of the fitting. In this case the measured values of the decay constants $f_{\pi}$ and $f_{K}$ are not used. However, it was shown that using the measured values $f_{\pi}$ significantly improves the convergence of the chiral expansion in the studies of the $\pi \pi(I=2)$ scattering lengths in Refs. [12,13]. Motivated from these studies, we adopt the chiral analysis with the measured values in the present work.

Before showing the results of the chiral analysis, we explain the decay constant used in the present work. The values of $f_{\pi}$ and $f_{K}$ in the same lattice setup have been calculated in Ref. [25]. They are defined in the normalization with $f_{\pi}=0.130 \mathrm{GeV}$ at the physical point and evaluated with the perturbative renormalization factor $Z_{A}^{\mathrm{P}}$. In the present work, we convert them to the values in the normalization with $f_{\pi}=0.092 \mathrm{GeV}$, and also to the values evaluated with the nonperturbative renormalization factor $Z_{A}^{\mathrm{NP}}$ by multiplying $Z_{A}^{\mathrm{NP}} /\left(u_{0} Z_{A}^{\mathrm{P}}\right)$. Here, $u_{0} \equiv P^{1 / 4}$ is the correction factor of the tadpole improved renormalization with plaquette value $P$ and takes $u_{0}=0.86968135$ (Ref. [34]). The renormalization factors are given by $Z_{A}^{\mathrm{P}}=$ 0.94279347 in Refs. [35-37] and $Z_{A}^{\mathrm{NP}}=0.781(20)$ in Ref. [34]. Converted values of $f_{\pi}$ and $f_{K}$ used in the present work are listed in Table VIII. Here, the systematic uncertainty for the determination of the renormalization factor $Z_{A}^{\mathrm{NP}}$ is added to the statistical errors in quadrature.

Here, we show the results of the analysis with the ChPT formulas of Eqs. (37), (38), (39), and (40). In the fitting,

TABLE VIII. The values of $f_{\pi}$ and $f_{K}$ used in the present work.

\begin{tabular}{lccccc}
\hline \hline$m_{\pi}[\mathrm{GeV}]$ & 0.17 & 0.30 & 0.41 & 0.57 & 0.71 \\
\hline$f_{\pi}[\mathrm{GeV}]$ & $0.0969(57)$ & $0.1030(29)$ & $0.1105(29)$ & $0.1260(42)$ & $0.1327(38)$ \\
$f_{K}[\mathrm{GeV}]$ & $0.1148(35)$ & $0.1195(32)$ & $0.1246(33)$ & $0.1353(41)$ & $0.1392(40)$ \\
\hline \hline
\end{tabular}


Data Set A
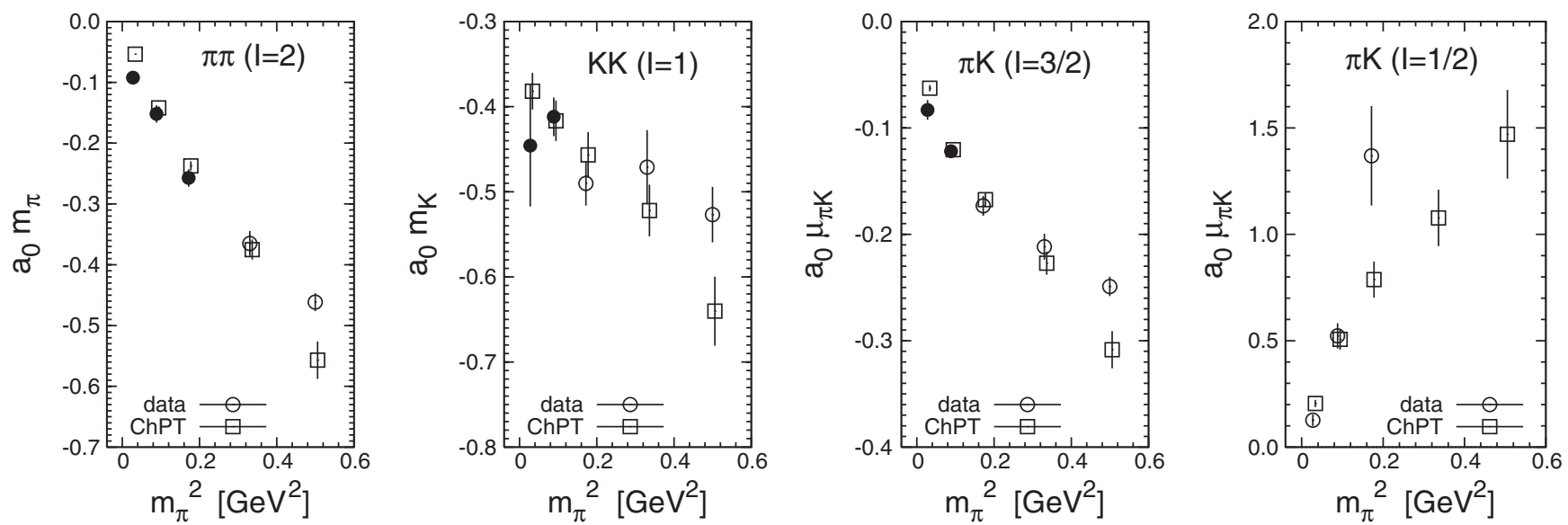

\section{Data Set B}
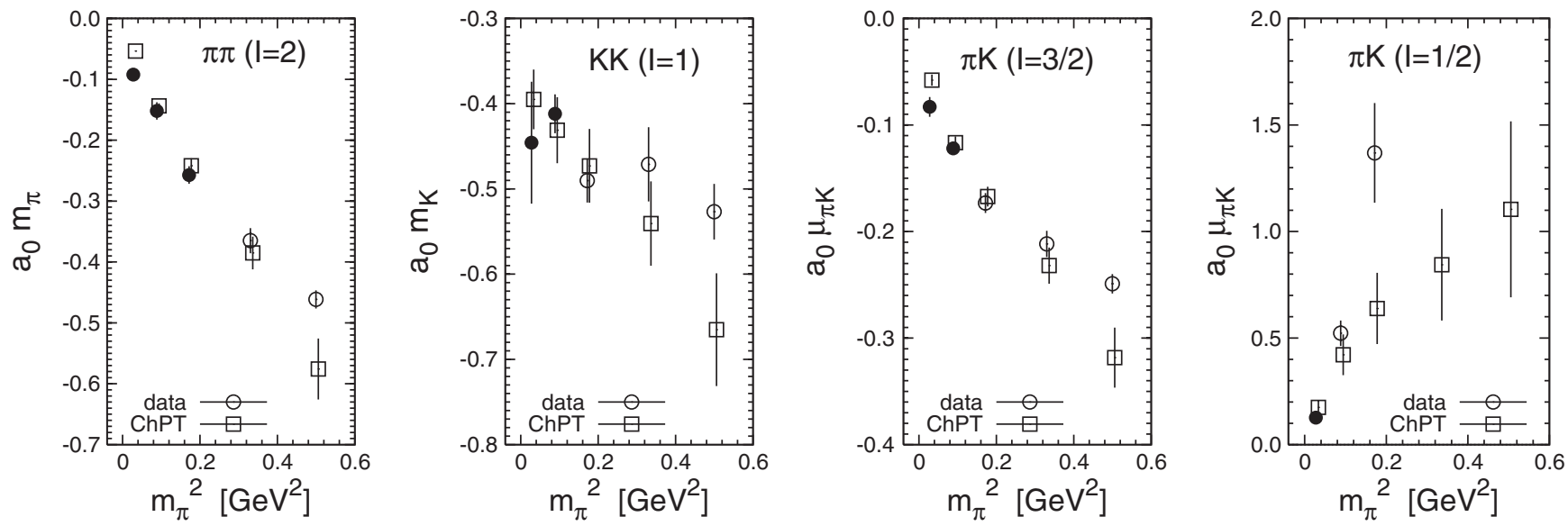

\section{Data Set C}
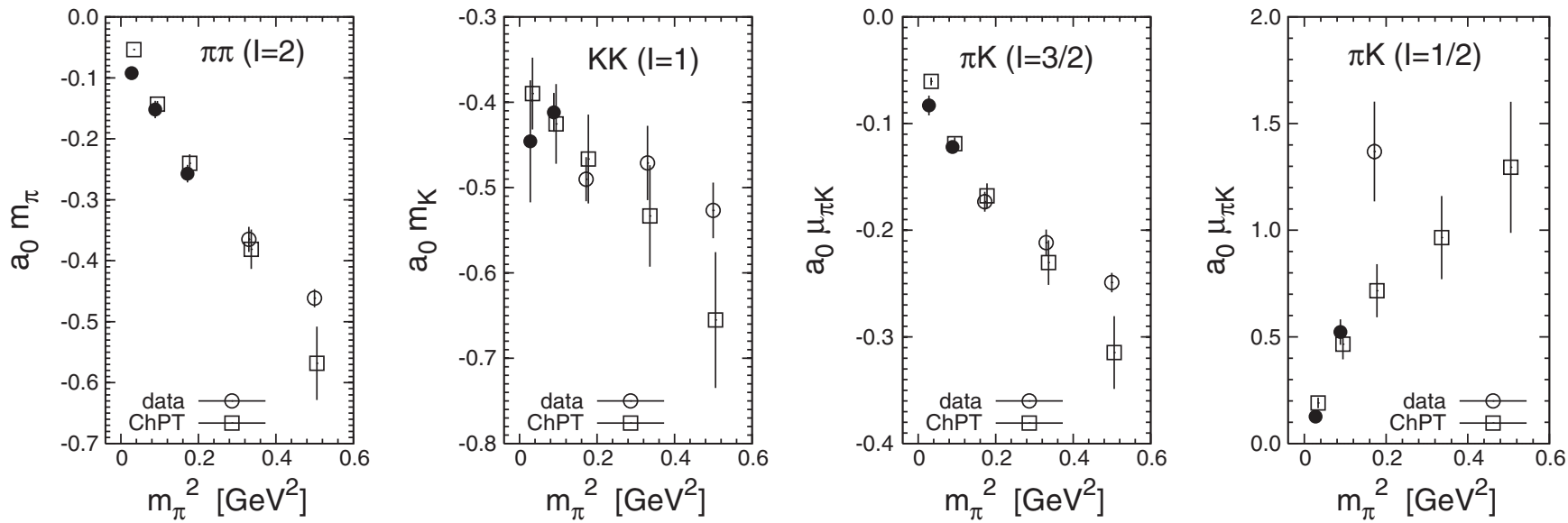

FIG. 9. Fitting results of the $\mathcal{O}\left(p^{4}\right)$ ChPT fit with the data sets $\mathrm{A}, \mathrm{B}$, and $\mathrm{C}$. The data are represented by circles, and those used in the fit by filled symbols. The fitting results by the ChPT formulas are denoted by squares. 
correlations among the scattering lengths for the the different channels are taken into account by the covariance matrix among them. The statistical errors of the fitting results of LECs are evaluated by the jackknife method. The errors of $f_{\pi}$ and $f_{K}$ are not included. The systematic error from the uncertainty for $f_{\pi}$ and $f_{K}$ is discussed in Sec. V B 3.

It was found in Ref. [25] that the formulas of $\mathcal{O}\left(p^{4}\right)$ ChPT describe the quark-mass dependence for $m_{\pi}, m_{K}$, and $f_{\pi}$ in $m_{\pi} \leq 0.41 \mathrm{GeV}$, and $f_{K}$ in $m_{\pi} \leq 0.30 \mathrm{GeV}$ well. From this, we consider that the formula of $\mathcal{O}\left(p^{4}\right)$ ChPT can be safely applied to our scattering length in these mass ranges. In the present work, for the repulsive channels, we analyze the following data:

$$
\begin{aligned}
a_{0}^{(2)} m_{\pi} & \text { for } m_{\pi}=0.17,0.30,0.41 \mathrm{GeV}, \\
a_{0}^{(1)} m_{K} & \text { for } m_{\pi}=0.17,0.30 \mathrm{GeV}, \\
a_{0}^{(3 / 2)} \mu_{\pi K} & \text { for } m_{\pi}=0.17,0.30 \mathrm{GeV}
\end{aligned}
$$

For the $\pi K(I=1 / 2)$ system, in the continuum theory, it is known that the convergence of the ChPT is not good compared with those for the repulsive channel. Thus, we need to test the convergence of the ChPT formula in this channel. In the present work, we consider the following three data sets of $a_{0}^{(1 / 2)} \mu_{\pi K}$ with the data in Eq. (42) for the chiral analysis, and investigate the stability of the fitting.

$$
\begin{aligned}
& \text { data set } \mathrm{A}: \text { not data, } \\
& \text { data set } \mathrm{B}: m_{\pi}=0.17 \mathrm{GeV}, \\
& \text { data set } \mathrm{C}: m_{\pi}=0.17,0.30 \mathrm{GeV},
\end{aligned}
$$

where these data sets are called data sets A, B, and C.

In Fig. 9, we plot the fitting results of the ChPT formulas with the data sets $\mathrm{A}, \mathrm{B}$, and $\mathrm{C}$. In all the cases, the ChPT formulas reproduce the data for $a_{0}^{(2)} m_{\pi}$ in $m_{\pi}=0.30$, $0.41 \mathrm{GeV}, a_{0}^{(1)} m_{K}, a_{0}^{(3 / 2)} \mu_{\pi K}$, and $a_{0}^{(1 / 2)} \mu_{\pi K}$ at $m_{\pi}=$ $0.30 \mathrm{GeV}$ well. At $m_{\pi}=0.17 \mathrm{GeV}$, however, the fitting results for some channels are not consistent with the data points. The deviation between the data and the fitting results can be also seen in the values of $\chi^{2} / N_{\text {d.o.f. }}\left(N_{\text {d.o.f. }}\right.$ is the degrees of freedom in the fit), which are plotted in Fig. 10 together with results of LECs $\left(10^{3} \cdot L_{5}\right.$ and $\left.10^{3} \cdot L^{\prime}\right)$. For each data set, $\chi^{2} / N_{\text {d.o.f. takes a huge number, }}$ $\mathcal{O}(10)$. This shows that the fitting with the $\mathcal{O}\left(p^{4}\right)$ ChPT formulas in Eqs. (37)-(40) does not work for our results of the scattering length.

\section{B. Chiral analysis with $\mathcal{O}\left(p^{4}\right)$ WChPT}

The scattering length vanishes in the chiral limit due to the chiral symmetry. But, for the Wilson fermion, it does not vanish due to the explicit chiral symmetry breaking from the Wilson term. We consider that an effect of this symmetry breaking causes the discrepancy between the our data and the formulas of ChPT. In order to investigate this, we need to consider the ChPT including the effect of the explicit chiral symmetry breaking, which has been proposed in Refs. [38-44], and usually called the WChPT. The WChPT formula for the $a_{0}^{(2)} m_{\pi}$ has been given by Ref. [45] in the case of $N_{\mathrm{f}}=2$. Here, we extend it to the WChPT formulas for the other scattering systems in the case of $N_{\mathrm{f}}=2+1$.

When we apply the WChPT to a chiral analysis, we choose an appropriate order counting rule for the quark mass $m_{\mathrm{q}}$ and the lattice cutoff $a$ for our lattice data. Our calculations are done with the nonperturbatively $\mathcal{O}(a)$ improved theory; thus, we treat only the terms higher than $\mathcal{O}\left(a^{2}\right)$ in the Lagrangian. In the present work, we adopt the following counting rule:

\section{CR1}

$$
\begin{gathered}
\text { LO }: p^{2}, m_{\mathrm{q}} \\
\text { NLO: } p^{4}, p^{2} m_{\mathrm{q}}, m_{\mathrm{q}}^{2}, a^{2} .
\end{gathered}
$$

In the following, we call this counting rule CR1. A dependence of the choice of the counting rule will be discussed in the next section.

In this counting rule, the scattering lengths for the $\pi \pi(I=2), K K(I=1), \pi K(I=3 / 2)$, and $\pi K(I=1 / 2)$ systems are given by

$$
\begin{gathered}
a_{0}^{(2)} m_{\pi}=\left.a_{0}^{(2)} m_{\pi}\right|_{\mathrm{ChPT}}-\frac{c_{2} a^{2}}{16 \pi f_{\pi}^{2}}, \\
a_{0}^{(1)} m_{K}=\left.a_{0}^{(1)} m_{K}\right|_{\mathrm{ChPT}}-\frac{c_{2} a^{2}}{16 \pi f_{K}^{2}}, \\
a_{0}^{(3 / 2)} \mu_{\pi K}=\left.a_{0}^{(3 / 2)} \mu_{\pi K}\right|_{\mathrm{ChPT}}-\frac{c_{2} a^{2}}{8 \pi f_{\pi} f_{K}} \cdot \frac{\mu_{\pi K}^{2}}{m_{\pi} m_{K}},
\end{gathered}
$$

FIG. 10. $\chi^{2} / N_{\text {d.o.f. }}$ and LECs $\left(10^{3} \cdot L_{5}\right.$ and $\left.10^{3} \cdot L^{\prime}\right)$ determined in the $\mathcal{O}\left(p^{4}\right)$ ChPT fit with the data sets A, B and C. 


\section{Data Set A}
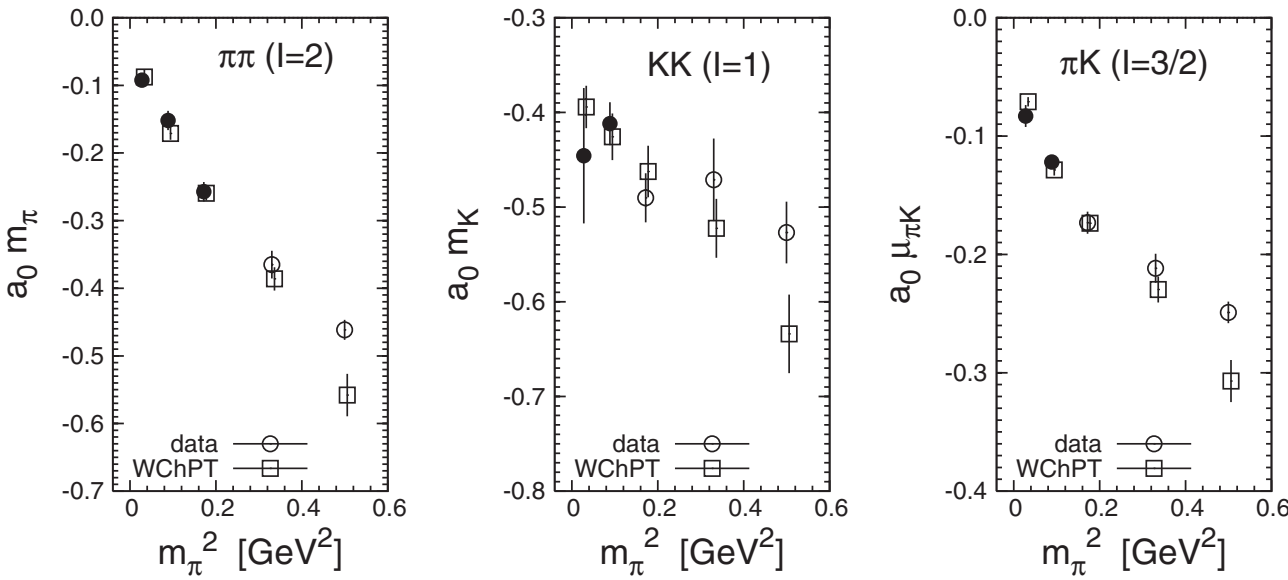

Data Set B
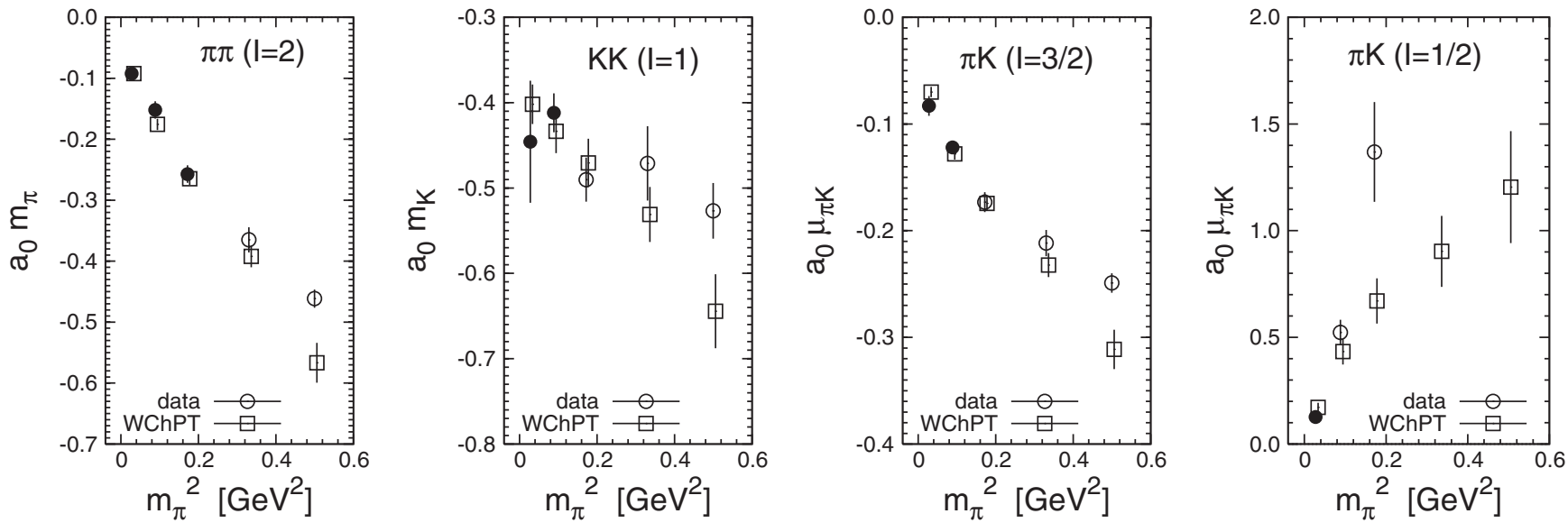

\section{Data Set C}
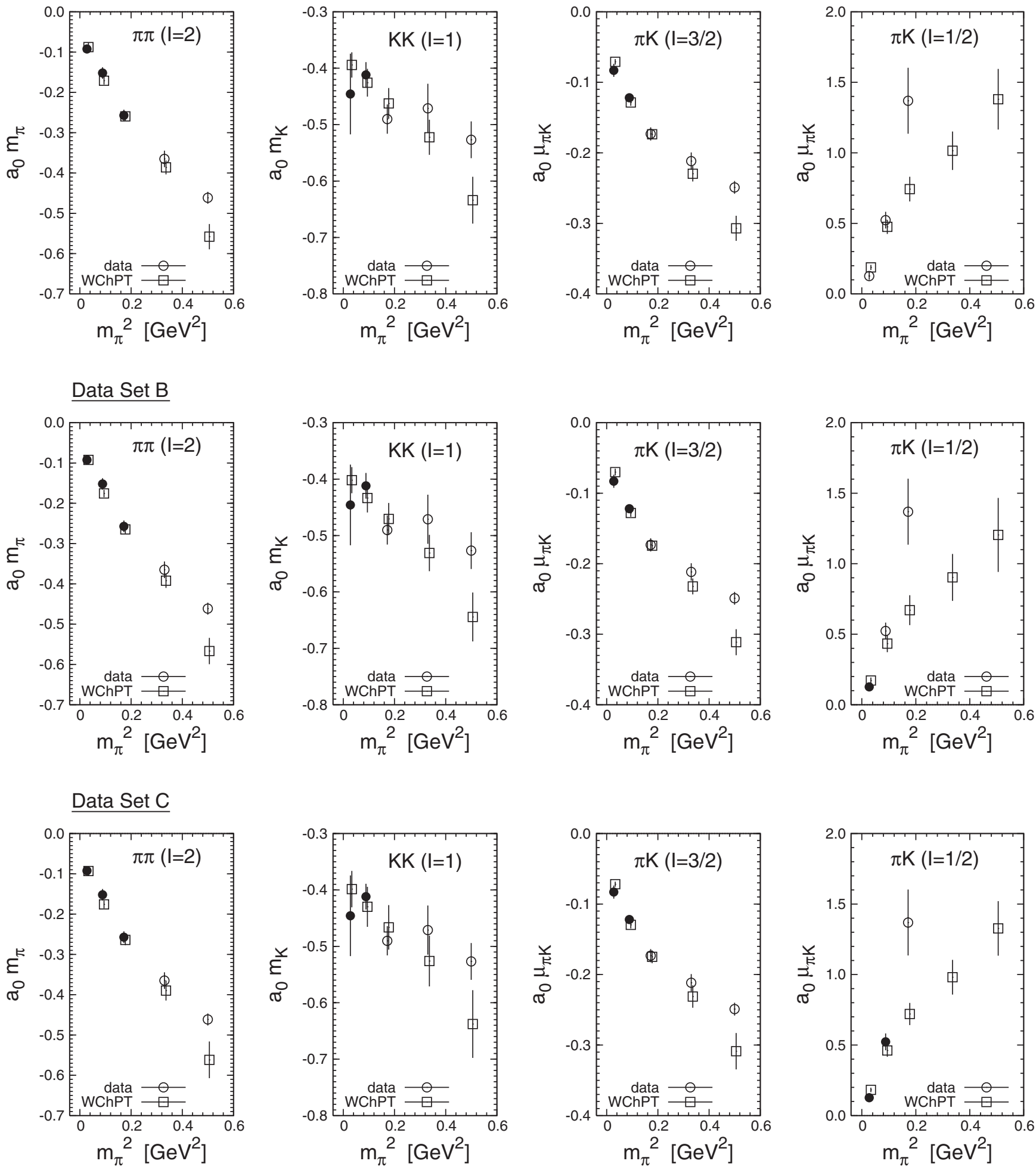

FIG. 11. Fitting results of the $\mathcal{O}\left(p^{4}\right)$ WChPT fit with the data sets A, B, and C. The data are represented by circles, and those used in the fit especially, are denoted by filled symbols. The fitting results by the WChPT formulas are denoted by squares. 


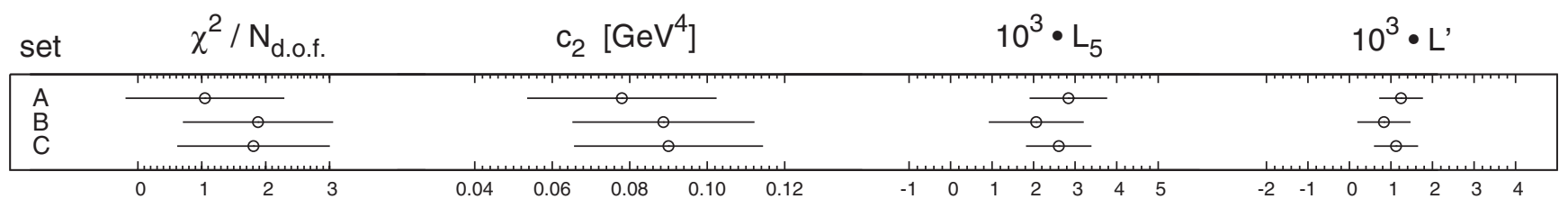

FIG. 12. $\chi^{2} / N_{\text {d.o.f. }}$ and LECs $\left(c_{2}, 10^{3} \cdot L_{5}\right.$, and $\left.10^{3} \cdot L^{\prime}\right)$ determined in the $\mathcal{O}\left(p^{4}\right)$ WChPT fit with the data sets A, B, and C.

$$
a_{0}^{(1 / 2)} \mu_{\pi K}=\left.a_{0}^{(1 / 2)} \mu_{\pi K}\right|_{\mathrm{ChPT}}-\frac{c_{2} a^{2}}{8 \pi f_{\pi} f_{K}} \cdot \frac{\mu_{\pi K}^{2}}{m_{\pi} m_{K}},
$$

where $\left.\quad a_{0}^{(2)} m_{\pi}\right|_{\mathrm{ChPT}},\left.\quad a_{0}^{(1)} m_{K}\right|_{\mathrm{ChPT}},\left.\quad a_{0}^{(3 / 2)} \mu_{\pi K}\right|_{\mathrm{ChPT}}, \quad$ and $\left.a_{0}^{(1 / 2)} \mu_{\pi K}\right|_{\text {ChPT }}$ are the ChPT formulas given by Eqs. (37), (38), (39), and (40), respectively. $c_{2}$ is a LEC of the WChPT. The details of these formulas are discussed in the Appendix.

Like as for the ChPT fit, we fix $m_{\pi}, m_{K}, f_{\pi}$, and $f_{K}$ in the WChPT formulas, the measured values by the lattice calculations at each quark mass. We fit our results with the formulas regarding the LECs $\left(L_{5}, L^{\prime}\right.$, and $\left.c_{2}\right)$ as free parameters. In Fig. 11, we plot the fitting results of the WChPT formulas with the data sets A, B, and C. We find that the fitting results at $m_{\pi}=0.17 \mathrm{GeV}$ are consistent with the data points. We show more detailed information in Fig. 12 , where $\chi^{2} / N_{\text {d.o.f. }}$ and LECs $\left(c_{2}, 10^{3} \cdot L_{5}\right.$, and $10^{3} \cdot L^{\prime}$ ) are given. $\chi^{2} / N_{\text {d.o.f. }}$ is improved compared with the ChPT fitting, and takes the reasonable value within the statistical error. We find that the fittings for three data sets give consistent results. This means that the WChPT formula works well for $a_{0}^{(2)}$ for $m_{\pi} \leq 0.41 \mathrm{GeV}, a_{0}^{(1)}$ and $a_{0}^{(3 / 2)}$ for $m_{\pi} \leq 0.30 \mathrm{GeV}$, and $a_{0}^{(1 / 2)}$ for $m_{\pi} \leq 0.30 \mathrm{GeV}$.

To investigate the convergence of the WChPT formulas, we consider the ratio of the next-to-leading terms to the leading term in Eqs. (45), (46), (47), and (48). In Fig. 13, we show
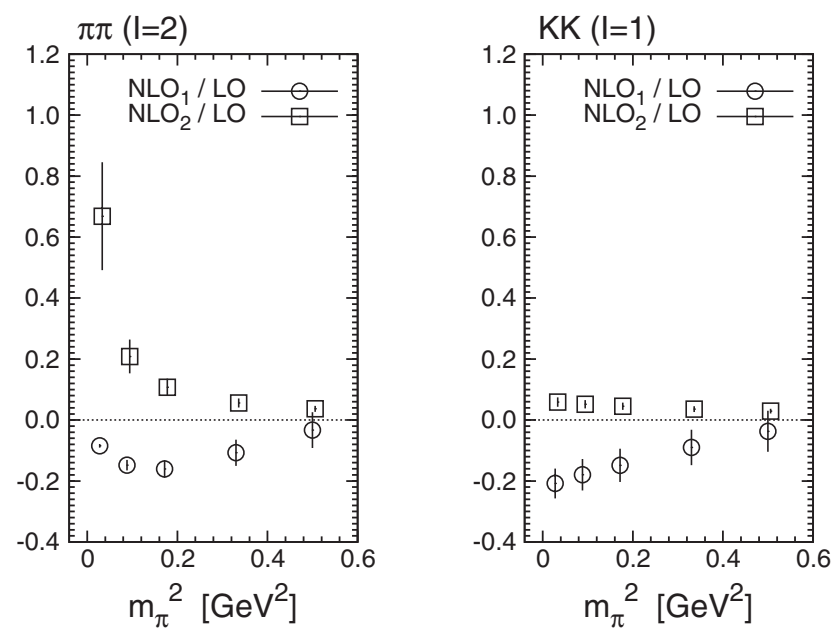

$$
\begin{aligned}
\frac{\mathrm{NLO}_{1}}{\mathrm{LO}} & =-\frac{16}{f_{\pi} f_{K}}\left[m_{\pi} m_{K} \cdot L^{\prime}-\frac{m_{\pi}^{2}+m_{K}^{2}}{4} \cdot L_{5}+\zeta^{(3 / 2)}\right], \\
\frac{\mathrm{NLO}_{2}}{\mathrm{LO}} & =\frac{c_{2} a^{2}}{m_{\pi} m_{K}}
\end{aligned}
$$

$$
\begin{aligned}
\frac{\mathrm{NLO}_{1}}{\mathrm{LO}} & =\frac{8}{f_{\pi} f_{K}}\left[m_{\pi} m_{K} \cdot L^{\prime}+2 \frac{m_{\pi}^{2}+m_{K}^{2}}{4} \cdot L_{5}+\zeta^{(1 / 2)}\right], \\
\frac{\mathrm{NLO}_{2}}{\mathrm{LO}} & =-\frac{c_{2} a^{2}}{2 m_{\pi} m_{K}}
\end{aligned}
$$

for the $\pi \pi(I=2), \quad K K(I=1), \quad \pi K(I=3 / 2), \quad$ and $\pi K(I=1 / 2)$ systems, respectively. In this figure, we use the LECs $\left(L^{\prime}, L_{5}\right.$, and $\left.c_{2}\right)$ obtained with data set B. For the repulsive channels, the ratios are at most $20 \%$
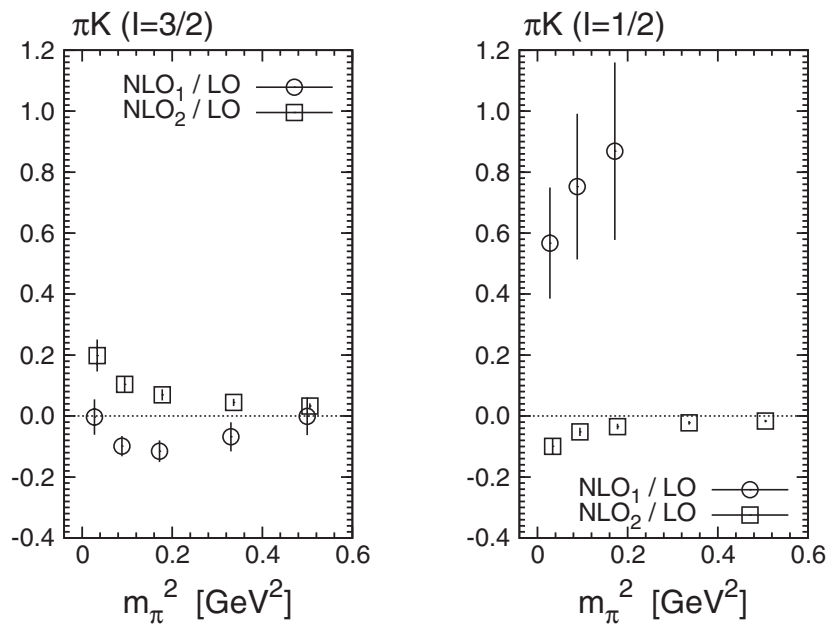

FIG. 13. The ratio of the next-to-leading terms to the leading term. $\mathrm{NLO}_{1} / \mathrm{LO}$ (circles) and $\mathrm{NLO}_{2}$ (squares) are represented. 
except for $\mathrm{NLO}_{2} / \mathrm{LO}$ of $a_{0}^{(2)} m_{\pi}$ at $m_{\pi}=0.17 \mathrm{GeV}$. The irregular $\mathrm{NLO}_{2} / \mathrm{LO}$ means that the effect of the explicit chiral symmetry breaking from the Wilson term cannot be negligible compared to the leading term of the WChPT for $a_{0}^{(2)} m_{\pi}$ at $m_{\pi}=0.17 \mathrm{GeV}$. For the $\pi K(I=1 / 2)$ system, we observe that $\mathrm{NLO}_{1} / \mathrm{LO}$ is not so small over a wide range of $m_{\pi}^{2}$. The convergence for the $\pi K(I=1 / 2)$ system might be disputable although the WChPT fit seems to work well from the point of view of $\chi^{2} / N_{\text {d.o.f. }}$. However, the number of data points is insufficient to perform the detailed investigation with $\mathcal{O}\left(p^{6}\right)$ WChPT fit. In the present work, alternatively, we discuss rough estimations of the $\mathcal{O}\left(p^{6}\right)$ contributions in Sec. V B 4.

\section{EXTRAPOLATION TO THE PHYSICAL POINT}

\section{A. Scattering lengths at the physical point}

We obtain the scattering length at the physical point by using the $\mathcal{O}\left(p^{4}\right)$ ChPT formulas, Eqs. (37)-(40), with the LECs $\left(L_{5}\right.$ and $\left.L^{\prime}\right)$ obtained from the $\mathcal{O}\left(p^{4}\right)$ WChPT fit in Sec. IVB. Here, at the physical point, $m_{\pi}=0.140 \mathrm{GeV}, \quad m_{K}=0.494 \mathrm{GeV}, \quad f_{\pi}=0.092 \mathrm{GeV}$, and $f_{K}=0.110 \mathrm{GeV}$. The results obtained with data sets $\mathrm{A}, \mathrm{B}$, and $\mathrm{C}$ are listed in Table IX.

As mentioned in Sec. IV B, three data sets give consistent results, and thus we adopt data set B for the standard fit. The extrapolated results including the systematic error are summarized as

$$
\begin{aligned}
a_{0}^{(2)} m_{\pi} & =-0.04263(22)(41), \\
a_{0}^{(1)} m_{K} & =-0.310(17)(32), \\
a_{0}^{(3 / 2)} \mu_{\pi K} & =-0.0469(24)(20), \\
a_{0}^{(1 / 2)} \mu_{\pi K} & =0.142(14)(27),
\end{aligned}
$$

TABLE IX. $\chi^{2} / N_{\text {d.o.f. }}$ and LECs obtained from the $\mathcal{O}\left(p^{4}\right)$ $S U$ (3) WChPT fits for data sets A, B, and C. The scattering lengths at the physical point are also shown.

\begin{tabular}{lccc}
\hline \hline Data set & $\mathrm{A}$ & $\mathrm{B}$ & $\mathrm{C}$ \\
\hline$\chi^{2} / N_{\text {d.o.f. }}$ & $1.1(1.2)$ & $1.9(1.2)$ & $1.8(1.2)$ \\
$c_{2}\left[\mathrm{GeV}^{4}\right]$ & $0.078(24)$ & $0.089(24)$ & $0.090(24)$ \\
$10^{3} \cdot L_{5}$ & $2.84(93)$ & $2.1(1.1)$ & $2.61(79)$ \\
$10^{3} \cdot L^{\prime}$ & $1.24(53)$ & $0.83(64)$ & $1.12(53)$ \\
\hline$a_{0}^{(2)} m_{\pi}$ & $-0.04259(21)$ & $-0.04263(22)$ & $-0.04260(30)$ \\
\hline$a_{0}^{(1)} m_{K}$ & $-0.307(16)$ & $-0.310(17)$ & $-0.308(23)$ \\
$a_{0}^{(1)} m_{\pi}$ & $-0.0871(45)$ & $-0.0880(47)$ & $-0.0872(65)$ \\
\hline$a_{0}^{(3 / 2)} \mu_{\pi K}$ & $-0.0486(20)$ & $-0.0469(24)$ & $-0.0481(14)$ \\
$a_{0}^{(3 / 2)} m_{\pi}$ & $-0.0623(26)$ & $-0.0602(31)$ & $-0.0617(18)$ \\
\hline$a_{0}^{(1 / 2)} \mu_{\pi K}$ & $0.152(12)$ & $0.142(14)$ & $0.149(10)$ \\
$a_{0}^{(1 / 2)} m_{\pi}$ & $0.195(15)$ & $0.183(18)$ & $0.191(13)$ \\
\hline \hline
\end{tabular}

where the first parenthesis is the statistical error and the second parenthesis represents the systematic error which is discussed in the following subsections.

\section{B. Estimate of systematic errors}

\section{Choice of the counting rule for the WChPT}

In this section, we investigate the dependence of the choice of the order counting rule for the results of the chiral analysis. Here, we consider another counting rule [counting rule 2 (CR2)],

\section{CR2}

$$
\begin{aligned}
\text { LO }: & p^{2}, m_{\mathrm{q}} \\
\text { NLO }: & a^{2} \\
\text { NNLO }: & p^{4}, p^{2} m_{\mathrm{q}}, m_{\mathrm{q}}^{2}, a^{3},
\end{aligned}
$$

and compare the results with CR2 to those with CR1 given in the previous section. The CR2 corresponds to $m_{\mathrm{q}} \simeq a^{3 / 2} \Lambda_{\mathrm{QCD}}^{5 / 2}(=6.7) \mathrm{MeV}$ with $a^{-1}=2.19 \mathrm{GeV}$ and $\Lambda_{\mathrm{QCD}}=0.217$ (24) GeV in the $\overline{\mathrm{MS}}$ scheme [46], while the CR1 does to $m_{\mathrm{q}} \simeq a \Lambda_{\mathrm{QCD}}^{2}(=22) \mathrm{MeV}$. The quarkmass parameters corresponding to $m_{\pi}=0.17,0.30,0.41$, $0.57,0.71 \mathrm{GeV}$ gives $m_{\mathrm{ud}}^{\overline{\mathrm{MS}}}=3.5,12,24,46,67 \mathrm{MeV}$, respectively [25]. For the data in $m_{\pi} \leq 0.41 \mathrm{GeV}$, which are used in our chiral analysis, it is not clear which counting rule is appropriate from these rough estimations. We need the quantitative comparison for the choice of the counting rule.

Because of the $\mathcal{O}\left(a^{3}\right)$ terms, the WChPT formulas given by Eqs. (45)-(48) are changed to

$$
\begin{aligned}
a_{0}^{(2)} m_{\pi}= & \left.a_{0}^{(2)} m_{\pi}\right|_{\mathrm{ChPT}}-\left(c_{2}+c_{3} \cdot \frac{a}{f_{\pi}^{2}}\right) \cdot \frac{a^{2}}{16 \pi f_{\pi}^{2}}, \\
a_{0}^{(1)} m_{K}= & \left.a_{0}^{(1)} m_{K}\right|_{\mathrm{ChPT}}-\left(c_{2}+c_{3} \cdot \frac{a}{f_{K}^{2}}\right) \cdot \frac{a^{2}}{16 \pi f_{K}^{2}}, \\
a_{0}^{(3 / 2)} \mu_{\pi K}= & \left.a_{0}^{(3 / 2)} \mu_{\pi K}\right|_{\mathrm{ChPT}} \\
& -\left(c_{2}+c_{3} \cdot \frac{a}{f_{\pi} f_{K}}\right) \cdot \frac{a^{2}}{8 \pi f_{\pi} f_{K}} \cdot \frac{\mu_{\pi K}^{2}}{m_{\pi} m_{K}}, \\
a_{0}^{(1 / 2)} \mu_{\pi K}= & \left.a_{0}^{(1 / 2)} \mu_{\pi K}\right|_{\mathrm{ChPT}} \\
& -\left(c_{2}+c_{3} \cdot \frac{a}{f_{\pi} f_{K}}\right) \cdot \frac{a^{2}}{8 \pi f_{\pi} f_{K}} \cdot \frac{\mu_{\pi K}^{2}}{m_{\pi} m_{K}},
\end{aligned}
$$

with an additional free parameter $c_{3}$. Here, $\left.a_{0}^{(2)} m_{\pi}\right|_{\mathrm{ChPT}}$, $\left.a_{0}^{(1)} m_{K}\right|_{\mathrm{ChPT}},\left.a_{0}^{(3 / 2)} \mu_{\pi K}\right|_{\mathrm{ChPT}}$, and $\left.a_{0}^{(1 / 2)} \mu_{\pi K}\right|_{\mathrm{ChPT}}$ are the scattering length of the ChPT given by Eqs. (37)-(40), respectively. 


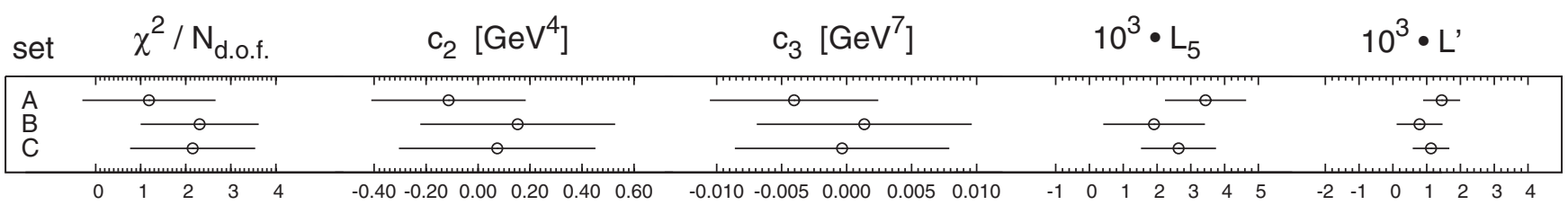

FIG. 14. $\chi^{2} / N_{\text {d.o.f. }}$ and LECs $\left(c_{2}, c_{3}, 10^{3} \cdot L_{5}\right.$, and $\left.10^{3} \cdot L^{\prime}\right)$ determined in the $\mathcal{O}\left(p^{4}\right)$ WChPT (CR2) fit with data sets A, B, and C.

In Fig. 14 , we show the results of the fitting. $\chi^{2} / N_{\text {d.o.f. }}$ takes the reasonable value within the statistical error. The scattering lengths at the physical point obtained with the data set A, B, and C are listed in Table X. For data set B, they are given as

$$
\begin{aligned}
a_{0}^{(2)} m_{\pi} & =-0.04258(40), \\
a_{0}^{(1)} m_{K} & =-0.306(30), \\
a_{0}^{(3 / 2)} \mu_{\pi K} & =-0.0464(42), \\
a_{0}^{(1 / 2)} \mu_{\pi K} & =0.141(18) .
\end{aligned}
$$

These are consistent with those obtained with the CR1 in Eq. (53) and the systematic error caused by the choice of the counting rule is negligible. Thus, we ignore the systematic error caused by the choice of the counting rule in the following discussion.

\section{Finite volume}

In this section, we discuss the systematic error of the finite volume, which appears from a deformation of the two-particle interaction due to the small lattice extent. For the $\pi \pi(I=2)$ system, the error has been estimated by the $\mathcal{O}\left(p^{4}\right) \quad S U(2) \quad$ ChPT. [47]. The contribution to $\left[\tan \delta_{0}(k) / k\right]^{-1}$ is considered to be the order of $\mathrm{e}^{-m_{\pi} L a}$. It is smaller than $6 \%$ of $\left[\tan \delta_{0}(k) / k\right]^{-1}$ at $m_{\pi}=0.14 \mathrm{GeV}$,

TABLE X. $\chi^{2} / N_{\text {d.o.f. }}$ and LECs obtained from the $\mathcal{O}\left(p^{4}\right)$ $S U$ (3) WChPT (CR2) fits for the data sets A, B, and C. The scattering lengths at the physical point are also shown.

\begin{tabular}{lccc}
\hline \hline Data set & $\mathrm{A}$ & $\mathrm{B}$ & $\mathrm{C}$ \\
\hline$\chi^{2} / N_{\text {d.o.f. }}$ & $1.2(1.5)$ & $2.3(1.3)$ & $2.2(1.4)$ \\
$c_{2}\left[\mathrm{GeV}^{4}\right]$ & $-0.11(30)$ & $0.15(37)$ & $0.07(38)$ \\
$c_{3}\left[\mathrm{GeV}^{7}\right]$ & $-0.0040(65)$ & $0.0014(83)$ & $-0.0003(82)$ \\
$10^{3} \cdot L_{5}$ & $3.4(1.2)$ & $1.9(1.5)$ & $2.6(1.1)$ \\
$10^{3} \cdot L^{\prime}$ & $1.44(55)$ & $0.79(68)$ & $1.13(54)$ \\
\hline$a_{0}^{(2)} m_{\pi}$ & $-0.04276(34)$ & $-0.04258(40)$ & $-0.04261(44)$ \\
\hline$a_{0}^{(1)} m_{K}$ & $-0.320(26)$ & $-0.306(30)$ & $-0.309(33)$ \\
$a_{0}^{(1)} m_{\pi}$ & $-0.0907(73)$ & $-0.0867(86)$ & $-0.0875(94)$ \\
\hline$a_{0}^{(3 / 2)} \mu_{\pi K}$ & $-0.0505(33)$ & $-0.0464(42)$ & $-0.0482(32)$ \\
$a_{0}^{(3 / 2)} m_{\pi}$ & $-0.0648(26)$ & $-0.0595(53)$ & $-0.0619(41)$ \\
\hline$a_{0}^{(1 / 2)} \mu_{\pi K}$ & $0.159(14)$ & $0.141(18)$ & $0.150(13)$ \\
$a_{0}^{(1 / 2)} m_{\pi}$ & $0.204(18)$ & $0.181(23)$ & $0.192(17)$ \\
\hline \hline
\end{tabular}

and $1 \%$ in $m_{\pi} \geq 0.29 \mathrm{GeV}$ at $L a=2.9 \mathrm{fm}$. It is much smaller than our statistical errors. In the $S U(3)$ case, we need to consider the contributions due to the $K$ and $\eta$ meson. However, they are considered to be smaller than the contribution from the pion. Thus, we ignore this systematic error in the following discussion.

\section{Uncertainty for $f_{\pi}$ and $f_{K}$}

We discuss the effects of the statistical uncertainty for the decay constants $f_{\pi}$ and $f_{K}$ in the WChPT formulas in Eqs. (45)-(48). In the following estimation, we use data set B. In order to investigate the effects, we carry out the fitting with $\left(f_{\pi} \pm \sigma\left(f_{\pi}\right), f_{K} \pm \sigma\left(f_{K}\right)\right)$ with 1 standard deviation, $\sigma\left(f_{\pi}\right)$ and $\sigma\left(f_{K}\right)$, whose values are tabulated in Table VIII. We regard the maximum absolute values of the differences among these fit results as the systematic error from the uncertainty of the decay constants. We obtain

$$
\begin{aligned}
a_{0}^{(2)} m_{\pi} & =-0.04263(22) \pm 0.00032, \\
a_{0}^{(1)} m_{K} & =-0.310(17) \pm 0.024, \\
a_{0}^{(3 / 2)} \mu_{\pi K} & =-0.0469(24) \pm 0.0020, \\
a_{0}^{(1 / 2)} \mu_{\pi K} & =0.142(14) \pm 0.011,
\end{aligned}
$$

where the second terms are the systematic errors from the decay constants. We find that these errors are comparable with the statistical error.

\section{Higher order effects of ChPT}

In this section, we give rough estimations of contributions of the $\mathcal{O}\left(p^{6}\right)$ terms at the physical point. The $\mathcal{O}\left(p^{2}\right)$ and $\mathcal{O}\left(p^{4}\right)$ contributions of our results at the physical point are

$$
\begin{array}{ccc} 
& \mathcal{O}\left(p^{2}\right) & \mathcal{O}\left(p^{4}\right) \\
a_{0}^{(2)} m_{\pi}: & -0.04607 & -0.04263(22), \\
a_{0}^{(1)} m_{K}: & -0.401 & -0.310(17), \\
a_{0}^{(3 / 2)} \mu_{\pi K}: & -0.0468 & -0.0469(24), \\
a_{0}^{(1 / 2)} \mu_{\pi K}: & 0.0936 & 0.142(14) .
\end{array}
$$

We roughly estimate the pure $\mathcal{O}\left(p^{6}\right)$ contributions by $X^{\mathcal{O}\left(p^{2}\right)} \times\left(1-X^{\mathcal{O}\left(p^{4}\right)} / X^{\mathcal{O}\left(p^{2}\right)}\right)^{2}$ for $X=a_{0}^{(2)} m_{\pi}, \quad a_{0}^{(1)} m_{K}$, $a_{0}^{(3 / 2)} m_{\pi}, a_{0}^{(1 / 2)} m_{\pi}$. We regard them as the systematic error from an uncertainty of the higher order terms of ChPT. We obtain 
TABLE XI. $\quad a_{0}^{(2)} m_{\pi}, a_{0}^{(1)} m_{K}, a_{0}^{(3 / 2)} m_{\pi}$, and $a_{0}^{(1 / 2)} m_{\pi}$ in the present work are shown in a comparison with the previous works. As the previous works, we refer to the experimental value by E865 [48] and NA48/2 [49], the phenomenological evaluations by Colangelo et al. [50] and Büttiker et al. [51], and the lattice calculations by the NPLQCD Collaboration [12,15,18], the ETM Collaboration [13], Yagi et al. [14], and $\mathrm{Fu}[5,19]$. We note that for $a_{0}^{(2)} m_{\pi}$ and $a_{0}^{(1)} m_{K}$ of the NPLQCD Collaboration, the combined errors, where the statistical and systematic errors are added in quadrature, are listed.

\begin{tabular}{|c|c|c|c|c|}
\hline & $a_{0}^{(2)} m_{\pi}$ & $a_{0}^{(1)} m_{K}$ & $a_{0}^{(3 / 2)} m_{\pi}$ & $a_{0}^{(1 / 2)} m_{\pi}$ \\
\hline The present work & $-0.04263(22)(41)$ & $-0.310(17)(32)$ & $-0.0602(31)(26)$ & $0.183(18)(35)$ \\
\hline $\begin{array}{l}\text { E865 [48] } \\
\text { NA48/2 [49] }\end{array}$ & $\begin{array}{c}-0.0432(86) \\
-0.0447(7)\end{array}$ & & & \\
\hline $\begin{array}{l}\text { Colangelo et al. [50] } \\
\text { Büttiker et al. [51] }\end{array}$ & $-0.0444(10)$ & & $-0.0448(77)$ & $0.224(22)$ \\
\hline $\begin{array}{l}\text { NPLQCD }[12,15,18] \\
\text { ETM [13] } \\
\text { Yagi et al. }[14] \\
\text { Fu [5,19] }\end{array}$ & $\begin{array}{c}-0.04330(42) \\
-0.04385(28)(38) \\
-0.04410(69)(18) \\
-0.04430(25)(40)\end{array}$ & $-0.352(16)$ & $-0.0574(16)\left(\begin{array}{c}+24 \\
-58\end{array}\right)$ & $0.1725(13)\left(\begin{array}{c}+23 \\
-156\end{array}\right)$ \\
\hline
\end{tabular}

$$
\begin{aligned}
a_{0}^{(2)} m_{\pi} & =-0.04263(22) \pm 0.00026, \\
a_{0}^{(1)} m_{K} & =-0.310(17) \pm 0.021, \\
a_{0}^{(3 / 2)} \mu_{\pi K} & =-0.0469(24) \pm 0.0001, \\
a_{0}^{(1 / 2)} \mu_{\pi K} & =0.142(14) \pm 0.025,
\end{aligned}
$$

where the second term refers to the systematic error.

This systematic error for the higher order effect is added to the systematic error due to the error of $f_{\pi}$ and $f_{K}$ in quadrature, and we regard it as the total systematic error which is given by the second term of Eq. (53).

\section{Comparison with the previous studies at the physical point}

For the $\pi K$ channels, some of the previous works used the values of $a_{0}^{(3 / 2)} m_{\pi}$ and $a_{0}^{(1 / 2)} m_{\pi}$, instead of $a_{0}^{(3 / 2)} \mu_{\pi K}$ and $a_{0}^{(1 / 2)} \mu_{\pi K}$. For the comparison, we present these values of our results,

$$
\begin{aligned}
& a_{0}^{(3 / 2)} m_{\pi}=-0.0602(31)(26), \\
& a_{0}^{(1 / 2)} m_{\pi}=0.183(18)(35) .
\end{aligned}
$$

In Table XI and Fig. 15, we show $a_{0}^{(2)} m_{\pi}, a_{0}^{(1)} m_{K}$, $a_{0}^{(3 / 2)} m_{\pi}$, and $a_{0}^{(1 / 2)} m_{\pi}$ determined in the present work together with the previous works. As the previous works, we refer the experimental values by E865 [48] and NA48/2 [49], the phenomenological evaluations by Colangelo et al. [50], and Büttiker et al. [51], and the lattice calculations by the NPLQCD Collaboration [12,15,18], the ETM Collaboration [13], Yagi et al. [14], and $\mathrm{Fu}[5,19]$. In the figure, we do not plot the result of E865 due to the large statistical error. For the lattice calculations, we show the combined errors where the statistical and systematic errors are added in quadrature by dotted lines in addition to the statistical errors (solid lines).

Although all the lattice results in each channel are roughly consistent, there are the slight deviations from the previous works, especially in $a_{0}^{(2)} m_{\pi}$. The reason for the deviations is not clear at the present. For the quantitative understanding, the systematic study with the different lattice spacings near the physical point is needed in the future.

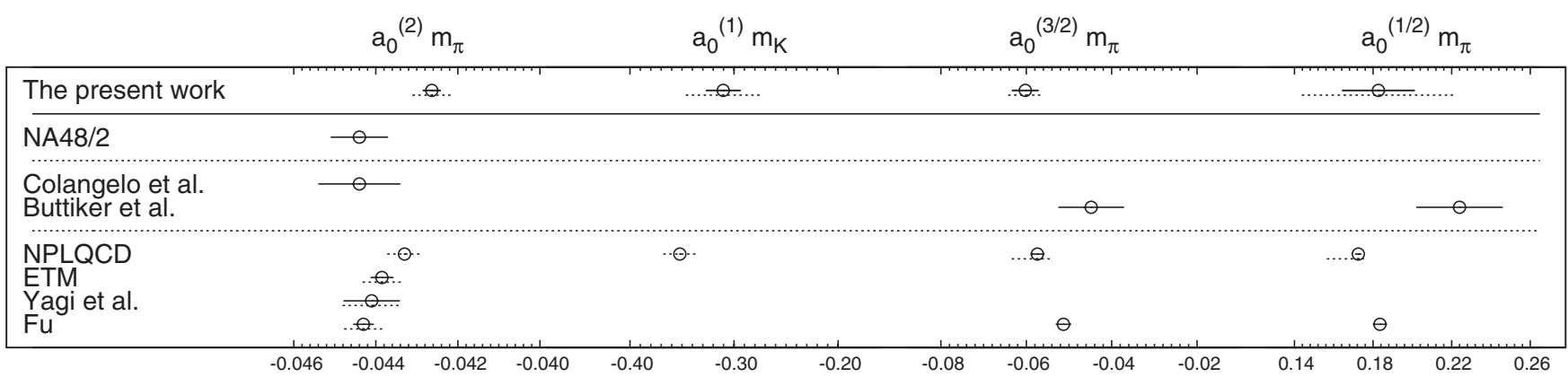

FIG. 15. $a_{0}^{(2)} m_{\pi}, a_{0}^{(1)} m_{K}, a_{0}^{(3 / 2)} m_{\pi}$, and $a_{0}^{(1 / 2)} m_{\pi}$ in the present work are shown in a comparison with the previous works. As the previous works, we refer the experimental value by NA48/2 [49], the phenomenological evaluations by Colangelo et al. [50] and Büttiker et al. [51], and the lattice calculations by the NPLQCD Collaboration [12,15,18], the ETM Collaboration [13], Yagi et al. [14], and $\mathrm{Fu}[5,19]$. 


\section{CONCLUSION}

The interaction of the $S$-wave two-meson systems $\quad[\pi \pi(I=2), \quad K K(I=1), \quad \pi K(I=3 / 2), \quad$ and $\pi K(I=1 / 2)]$ has been studied from lattice QCD. To reduce the computational cost, we have employed the method where one of the particles in the final state is fixed at a given time. For the $\pi K(I=1 / 2)$ system, we have used the variational method with the two operators to separate the contamination from the higher states. We have observed that the interaction at low energy is repulsive for the $\pi \pi(I=2), K K(I=1)$, and $\pi K(I=3 / 2)$ systems, and attractive for the $\pi K(I=1 / 2)$ system. This feature is consistent with the experiment.

The scattering lengths have been calculated by using Lüscher's finite size method. We have found that the attraction in the $\pi K(I=1 / 2)$ system becomes so strong in $m_{\pi}>0.41 \mathrm{GeV}$ that the sign of $\tan \delta_{0}(k) / k$ becomes negative. This fact indicates the formation of a bound state at heavy $m_{\pi}$ for the $\pi K(I=1 / 2)$ system. Therefore, we have used the data in $m_{\pi} \leq 0.30 \mathrm{GeV}$ to evaluate the reliable scattering length for this system.

We have investigated the quark-mass dependence of the scattering lengths to evaluate the values at the physical quark mass. For this purpose, we have considered the $\mathcal{O}\left(p^{4}\right)$ ChPT formulas. However, the fitting with these formulas does not work for our results of the scattering length, especially at $m_{\pi}=0.17 \mathrm{GeV}$. We alternatively have tried to fit with the $\mathcal{O}\left(p^{4}\right)$ WChPT formulas including the $\mathcal{O}\left(a^{2}\right)$ terms. We have found that these formulas reproduce the mass dependence of our results even near $m_{\pi}=0.17 \mathrm{GeV}$. The description seems to work well at least in $m_{\pi} \leq 0.41 \mathrm{GeV}$ for $a_{0}^{(2)} m_{\pi}$, in $m_{\pi} \leq 0.30 \mathrm{GeV}$ for $a_{0}^{(1)} m_{K}$ and $a_{0}^{(3 / 2)} \mu_{\pi K}$, and in $m_{\pi} \leq 0.30 \mathrm{GeV}$ for $a_{0}^{(1 / 2)} \mu_{\pi K}$. We have also discussed the possible systematic errors and evaluated the scattering lengths at the physical quark mass.

Although our lattice results are roughly consistent with the results of the previous studies, the deviations beyond the statistical error remain at the present. We need the systematic study with the different lattice spacings near the physical point for the quantitative understanding in the future.

\section{ACKNOWLEDGMENTS}

We thank colleagues in the PACS-CS Collaboration for helpful discussions and for providing us the code used in the present work. K. S. thanks Y. Namekawa for reading the manuscript carefully. This study is supported by Grants-in-Aid for Scientific Research on Priority Area (No. 21105506) from the Ministry of Education, Culture, Sports, Science, and Technology. The numerical calculations were carried out on the super parallel computers, PACS-CS and T2K-Tsukuba at the University of Tsukuba, and TSUBAME at the Tokyo Institute of Technology.

\section{APPENDIX: $\mathcal{O}\left(p^{4}\right) S U(3)$ WCHPT FORMULAS}

We give the formulas constructed from the $\mathcal{O}\left(p^{4}\right) S U(3)$ WChPT with the CR1. According to Ref.[41], the $\mathcal{O}\left(a^{2}\right)$ Lagrangian consists of three terms written as

$$
\begin{aligned}
\mathcal{L}_{\mathcal{O}\left(a^{2}\right)}= & w_{6} \cdot \frac{a^{2} F^{2}}{16} \cdot\left\langle U+U^{\dagger}\right\rangle^{2}+w_{7} \cdot \frac{a^{2} F^{2}}{16} \cdot\left\langle U-U^{\dagger}\right\rangle^{2} \\
& +w_{8} \cdot \frac{a^{2} F^{2}}{8} \cdot\left\langle U^{2}+\left(U^{\dagger}\right)^{2}\right\rangle
\end{aligned}
$$

in the Minkowski space-time, where $U=\mathrm{e}^{i \Phi / F}$ with the NG-boson field matrix $\Phi$, and the angle bracket means the trace for the flavor indices. $w_{6}, w_{7}$, and $w_{8}$ are the LECs in the $S U(3)$ WChPT. The LEC $c_{2}$ discussed in Sec. IV B is defined by $c_{2} \equiv-8 w_{6}-4 w_{8}$. After calculating the generating function with zero external fields, we regard it as an effective action of NG-boson fields according to Ref. [52]. On-shell quantities can be obtained from this effective action.

We represent the mass of the NG boson $P(=\pi, K, \eta)$ at the tree level by $M_{P}$. They are written as

$$
\begin{aligned}
M_{\pi}^{2} & =2 B m_{\mathrm{ud}}, \\
M_{K}^{2} & =B\left(m_{\mathrm{ud}}+m_{\mathrm{s}}\right), \\
M_{\eta}^{2} & =B\left(2 m_{\mathrm{ud}}+4 m_{\mathrm{s}}\right) / 3,
\end{aligned}
$$

with a parameter $B$, bare quark masses $m_{\mathrm{ud}}\left(\equiv m_{\mathrm{u}}=m_{\mathrm{d}}\right)$, and $m_{\mathrm{s}}$. It is useful to consider the shifted mass with the $\mathcal{O}\left(a^{2}\right)$ terms as

$$
\bar{M}_{P}^{2}=M_{P}^{2}+\left(12 w_{6}+4 w_{8}\right) a^{2}
$$

because the NG-boson mass always enters the WChPT Lagrangian with the form of $\bar{M}_{P}^{2}$.

The NG-boson masses up to the $\mathcal{O}\left(p^{4}\right)$ terms can be described as

$$
\begin{aligned}
m_{\pi}^{2}= & \bar{M}_{\pi}^{2}\left[1+\frac{\bar{M}_{\pi}^{2}}{F^{2}}\left(-8 L_{4}-8 L_{5}+16 L_{6}+16 L_{8}\right)\right. \\
& \left.+\frac{\bar{M}_{K}^{2}}{F^{2}}\left(-16 L_{4}+32 L_{6}\right)+\frac{\bar{\mu}_{\pi}}{F^{2}}-\frac{1}{3} \frac{\bar{\mu}_{\eta}}{F^{2}}\right],
\end{aligned}
$$

$$
\begin{aligned}
m_{K}^{2}= & \bar{M}_{K}^{2}\left[1+\frac{\bar{M}_{K}^{2}}{F^{2}}\left(-16 L_{4}-8 L_{5}+32 L_{6}+16 L_{8}\right)\right. \\
& \left.+\frac{\bar{M}_{\pi}^{2}}{F^{2}}\left(-8 L_{4}+16 L_{6}\right)+\frac{2}{3} \frac{\bar{\mu}_{\eta}}{F^{2}}\right],
\end{aligned}
$$

where $\bar{\mu}_{P}=\frac{1}{32 \pi^{2}} \bar{M}_{P}^{2} \log \left(\bar{M}_{P}^{2} / \mu^{2}\right)$. The difference from the continuum ChPT originates only from $\bar{M}_{P}^{2}$ in the leading order. It is noted that $\bar{M}_{\pi}^{4}, \bar{M}_{\pi}^{2} \bar{M}_{K}^{2}$ and $\bar{M}_{K}^{4}$ in the 
next-to-leading order are indistinguishable from $M_{\pi}^{4}$, $M_{\pi}^{2} M_{K}^{2}$, and $M_{K}^{4}$, respectively, up to $\mathcal{O}\left(p^{4}\right)$ terms.

The decay constant of the pion and kaon up to the $\mathcal{O}\left(p^{4}\right)$ terms can be described as

$$
\begin{gathered}
f_{\pi}=F\left[1+\frac{\bar{M}_{\pi}^{2}}{F^{2}}\left(4 L_{4}+4 L_{5}\right)+\frac{\bar{M}_{K}^{2}}{F^{2}}\left(8 L_{4}\right)-2 \frac{\bar{\mu}_{\pi}}{F^{2}}-\frac{\bar{\mu}_{K}}{F^{2}}\right], \\
f_{K}=F\left[1+\frac{\bar{M}_{K}^{2}}{F^{2}}\left(8 L_{4}+4 L_{5}\right)+\frac{\bar{M}_{\pi}^{2}}{F^{2}}\left(4 L_{4}\right)\right. \\
\left.-\frac{3}{4} \frac{\bar{\mu}_{\pi}}{F^{2}}-\frac{3}{2} \frac{\bar{\mu}_{K}}{F^{2}}-\frac{3}{4} \frac{\bar{\mu}_{\eta}}{F^{2}}\right] .
\end{gathered}
$$

The difference from the continuum ChPT does not exist because $\bar{M}_{\pi}^{2}$ and $\bar{M}_{K}{ }^{2}$ in the next-to-leading order are indistinguishable from $M_{\pi}^{2}$ and $M_{K}^{2}$, respectively, up to $\mathcal{O}\left(p^{4}\right)$ terms.

The scattering lengths of the $\pi \pi(I=2), K K(I=1)$, $\pi K(I=3 / 2)$, and $\pi K(I=1 / 2)$ systems are already given in Eqs. (45), (46), (47), and (48) with Eqs. (37), (38), (39), and (40) except for the definitions of $\zeta^{(2),(1),(3 / 2),(1 / 2)}$. They are written as

$$
\zeta^{(2)}=\frac{1}{(16 \pi)^{2}}\left[-\frac{3 m_{\pi}^{2}}{2} \log \left(\frac{m_{\pi}^{2}}{\mu^{2}}\right)-\frac{m_{\pi}^{2}}{18} \log \left(\frac{m_{\eta}^{2}}{\mu^{2}}\right)+\frac{4 m_{\pi}^{2}}{9}\right],
$$

where $t_{1}\left(m_{\pi}, m_{K}\right), t_{2}\left(m_{\pi}, m_{K}\right)$ can be written as

$$
\begin{aligned}
& t_{1}\left(m_{\pi}, m_{K}\right)=\frac{\sqrt{\left(m_{K}+m_{\pi}\right)\left(2 m_{K}-m_{\pi}\right)}}{m_{K}-m_{\pi}} \arctan \left(\frac{2\left(m_{K}-m_{\pi}\right)}{m_{K}+2 m_{\pi}} \sqrt{\frac{m_{K}+m_{\pi}}{2 m_{K}-m_{\pi}}}\right), \\
& t_{2}\left(m_{\pi}, m_{K}\right)=\frac{\sqrt{\left(m_{K}-m_{\pi}\right)\left(2 m_{K}+m_{\pi}\right)}}{m_{K}+m_{\pi}} \arctan \left(\frac{2\left(m_{K}+m_{\pi}\right)}{m_{K}-2 m_{\pi}} \sqrt{\frac{m_{K}-m_{\pi}}{2 m_{K}+m_{\pi}}}\right) .
\end{aligned}
$$

We used $\bar{M}_{P}^{2}=m_{P}^{2}, \bar{\mu}_{P}^{2}=\mu_{P}^{2}\left(\equiv \frac{1}{32 \pi^{2}} m_{P}^{2} \log \left(m_{P}^{2} / \mu^{2}\right)\right)$ and $F^{2}=f_{\pi}^{2}=f_{K}^{2}$ at the $\mathcal{O}\left(p^{4}\right)$ terms to simplify the expression. We also used the tree-level (Gell-Mann-Okubo) relation, $m_{\eta}^{2}=\left(4 m_{K}^{2}-m_{\pi}^{2}\right) / 3$ for the mass of the $\eta$ meson. These relations are sufficient if we restrict ourselves up to the $\mathcal{O}\left(p^{4}\right)$ expression. 
[1] M. Lüscher, Commun. Math. Phys. 105, 153 (1986); , Nucl. Phys. B354, 531 (1991).

[2] M. Fukugita, Y. Kuramashi, M. Okawa, H. Mino, and A. Ukawa, Phys. Rev. D 52, 3003 (1995).

[3] Q. Liu (RBC Collaboration and UKQCD Collaboration), Proc. Sci. LAT2009 (2009) 101 [arXiv:0910.2658].

[4] Z. Fu, Commun. Theor. Phys. 57, 78 (2012).

[5] Z. Fu, Phys. Rev. D 87, 074501 (2013).

[6] S. R. Sharpe, R. Gupta, and G. W. Kilcup, Nucl. Phys. B383, 309 (1992).

[7] S. Aoki et al. (JLQCD Collaboration), Phys. Rev. D 66, 077501 (2002).

[8] S. Aoki et al. (CP-PACS Collaboration), Phys. Rev. D 67, 014502 (2003).

[9] X. Li et al. (CLQCD Collaboration), J. High Energy Phys. 06 (2007) 053.

[10] T. Yamazaki et al. (CP-PACS Collaboration), Phys. Rev. D 70, 074513 (2004).

[11] S. R. Beane, P. Bedaque, K. Orginos, and M. Savage (NPLQCD Collaboration), Phys. Rev. D 73, 054503 (2006).

[12] S. R. Beane, T. Luu, K. Orginos, A. Parreño, M. Savage, A. Torok, and A. Walker-Loud (NPLQCD Collaboration), Phys. Rev. D 77, 014505 (2008).

[13] X. Feng, K. Jansen, and D. Renner (ETM Collaboration), Phys. Lett. B 684, 268 (2010).

[14] T. Yagi, S. Hashimoto, O. Morimatsu, and M. Ohtani, arXiv:1108.2970.

[15] S. R. Beane, T. Luu, K. Orginos, A. Parreño, M. Savage, A. Torok, and A. Walker-Loud (NPLQCD Collaboration), Phys. Rev. D 77, 094507 (2008).

[16] C. Miao, X. i. Du, G. w. Meng, and C. Liu, Phys. Lett. B 595, 400 (2004).

[17] J. Nagata, S. Muroya, and A. Nakamura, Phys. Rev. C 80, 045203 (2009).

[18] S. R. Beane, P. Bedaque, T. Luu, K. Orginos, E. Pallante, A. Parreño, and M. Savage (NPLQCD Collaboration), Phys. Rev. D 74, 114503 (2006).

[19] Z. Fu, Phys. Rev. D 85, 074501 (2012).

[20] C. B. Lang, L. Leskovec, D. Mohler, and S. Prelovsek, Phys. Rev. D 86, 054508 (2012).

[21] K. Sasaki et al. (PACS-CS Collaboration), Proc. Sci. LAT2009 (2009) 098 [arXiv:0911.0228]; K. Sasaki et al. (PACS-CS Collaboration), Prog. Theor. Phys. Suppl. 186, 187 (2010).

[22] M. Luscher, Commun. Math. Phys. 104, 177 (1986).

[23] S. Sasaki and T. Yamazaki, Phys. Rev. D 74, 114507 (2006).

[24] M. Lüscher and U. Wolff, Nucl. Phys. B339, 222 (1990).

[25] S. Aoki et al. (PACS-CS Collaboration), Phys. Rev. D 79, 034503 (2009).

[26] S. Aoki et al. (PACS-CS Collaboration), Phys. Rev. D 81, 074503 (2010).
[27] C. W. Bernard, T. Burch, K. Orginos, D. Toussaint, T. A. DeGrand, C. E. Detar, S. Datta, S. A. Gottlieb, U. M. Heller, J. E. Hetrick, and R. Sugar, Phys. Rev. D 64, 054506 (2001).

[28] C. Aubin, C. Bernard, C. DeTar, J. Osborn, S. Gottlieb, E. B. Gregory, D. Toussaint, U. M. Heller, J. E. Hetrick, and R. Sugar, Phys. Rev. D 70, 094505 (2004).

[29] A. Hart, C. McNeile, C. Michael, and J. Pickavance (UKQCD Collaboration), Phys. Rev. D 74, 114504 (2006).

[30] H. Suganuma, K. Tsumura, N. Ishii, and F. Okiharu, Prog. Theor. Phys. Suppl. 168, 168 (2007).

[31] J. Gasser and H. Leutwyler, Nucl. Phys. B250, 465 (1985).

[32] V. Bernard, N. Kaiser, and U. G. Meissner, Nucl. Phys. B357, 129 (1991).

[33] J. W. Chen, D. O'Connell, and A. Walker-Loud, Phys. Rev. D 75, 054501 (2007).

[34] S. Aoki et al. (PACS-CS Collaboration), J. High Energy Phys. 08 (2010) 101.

[35] S. Aoki, K.-i. Nagai, Y. Taniguchi, and A. Ukawa, Phys. Rev. D 58, 074505 (1998).

[36] Y. Taniguchi and A. Ukawa, Phys. Rev. D 58, 114503 (1998).

[37] S. Aoki, R. Frezzotti, and P. Weisz, Nucl. Phys. B540, 501 (1999).

[38] S. R. Sharpe and R. L. Singleton, Jr., Phys. Rev. D 58, 074501 (1998).

[39] G. Rupak and N. Shoresh, Phys. Rev. D 66, 054503 (2002).

[40] S. Aoki, Phys. Rev. D 68, 054508 (2003).

[41] O. Bär, G. Rupak, and N. Shoresh, Phys. Rev. D 70, 034508 (2004).

[42] S. R. Sharpe and J. M. S. Wu, Phys. Rev. D 71, 074501 (2005).

[43] S. Aoki, O. Bär, T. Ishikawa, and S. Takeda, Phys. Rev. D 73, 014511 (2006).

[44] M. T. Hansen and S. R. Sharpe, Phys. Rev. D 85, 054504 (2012).

[45] S. Aoki, O. Bar, and B. Biedermann, Phys. Rev. D 78, 114501 (2008).

[46] S. Eidelman et al. (Particle Data Group), Phys. Lett. B 592, 1 (2004).

[47] P. F. Bedaque, I. Sato, and A. Walker-Loud, Phys. Rev. D 73, 074501 (2006).

[48] S. Pislak, R. Appel, G. S. Atoyan, B. Bassalleck, D. R. Bergman, N. Cheung, S. Dhawan, and H. Do et al., Phys. Rev. D 67, 072004 (2003); 81, 119903(E) (2010).

[49] J. R. Batley et al. (NA48/2 Collaboration), Eur. Phys. J. C 70, 635 (2010).

[50] G. Colangelo, J. Gasser, and H. Leutwyler, Phys. Lett. B 488, 261 (2000).

[51] P. Buettiker, S. Descotes-Genon, and B. Moussallam, Eur. Phys. J. C 33, 409 (2004).

[52] J. Gasser and H. Leutwyler, Nucl. Phys. B250, 539 (1985). 